\title{
The Small GTPase RAC1/CED-10 Is Essential in Maintaining Dopaminergic Neuron Function and Survival Against a-Synuclein-Induced Toxicity
}

\author{
Hanna Kim ${ }^{1}$ - Carles Calatayud ${ }^{2,3,4}$. Sanjib Guha ${ }^{5}$ - Irene Fernández-Carasa ${ }^{2,3}$ - Laura Berkowitz ${ }^{1}$ • \\ Iria Carballo-Carbajal ${ }^{6}$ - Mario Ezquerra ${ }^{7}$ - Rubén Fernández-Santiago ${ }^{7}$ - Pankaj Kapahi ${ }^{5}$ - Ángel Raya ${ }^{4,8}$. \\ Antonio Miranda-Vizuete ${ }^{9} \cdot$ Jose Miguel Lizcano $^{10} \cdot$ Miquel Vila $^{6,8,10} \cdot \operatorname{Kim~A.~Caldwell~}^{1} \cdot$ Guy A. Caldwell $^{1}$. \\ Antonella Consiglio ${ }^{2,3,11}$. Esther Dalfo ${ }^{10,12}$ (D)
}

Received: 14 August 2017 / Accepted: 7 January 2018 / Published online: 10 February 2018

(C) The Author(s) 2018

\begin{abstract}
Parkinson's disease is associated with intracellular $\alpha$-synuclein accumulation and ventral midbrain dopaminergic neuronal death in the Substantia Nigra of brain patients. The Rho GTPase pathway, mainly linking surface receptors to the organization of the actin and microtubule cytoskeletons, has been suggested to participate to Parkinson's disease pathogenesis. Nevertheless, its exact contribution remains obscure. To unveil the participation of the Rho GTPase family to the molecular pathogenesis of Parkinson's disease, we first used C elegans to demonstrate the role of the small GTPase RAC1 (ced-10 in the worm) in maintaining dopaminergic function and survival in the presence of alpha-synuclein. In addition, ced-10 mutant worms determined an increase of alpha-synuclein inclusions in comparison to control worms as well as an increase in autophagic vesicles. We then used a human neuroblastoma cells (M17) stably over-expressing alpha-synuclein and found that $R A C 1$ function decreased the amount of amyloidogenic alpha-synuclein. Further, by using dopaminergic neurons derived from patients of familial LRRK2Parkinson's disease we report that human RAC1 activity is essential in the regulation of dopaminergic cell death, alpha-synuclein
\end{abstract}

Hanna Kim, Carles Calatayud and Sanjib Guha contributed equally to this work.

Electronic supplementary material The online version of this article (https://doi.org/10.1007/s12035-018-0881-7) contains supplementary material, which is available to authorized users.

Antonella Consiglio

consiglio@ub.edu

Esther Dalfo

esther.dalfo@uab.cat

1 Department of Biological Sciences, The University of Alabama, Tuscaloosa, AL 35487, USA

2 Department of Pathology and Experimental Therapeutics, Bellvitge University Hospital-IDIBELL, 08028 L'Hospitalet de Llobregat, Spain

3 Institute of Biomedicine of the University of Barcelona (IBUB), Barcelona 08908, Spain

4 Center of Regenerative Medicine in Barcelona (CMRB), Center for Networked Biomedical Research on Bioengineering, Biomaterials and Nanomedicine (CIBER-BBN), Hospital Duran i Reynals, 08908 L'Hospitalet de Llobregat, Spain

5 Buck Institute for Research on Aging, 8001 Redwood Boulevard, Novato, CA 94945, USA
6 Neurodegenerative Diseases Research Group, Vall d'Hebron Research Institute-Center for Networked Biomedical Research on Neurodegenerative Diseases (CIBERNED), 08035 Barcelona, Spain

7 Laboratory of Parkinson Disease and Other Neurodegenerative Movement Disorders, Department of Neurology: Clinical and Experimental Research, IDIBAPS - Hospital Clínic de Barcelona, 08036 Barcelona, Spain

8 Catalan Institution for Research and Advanced Studies (ICREA), 08010 Barcelona, Spain

9 Instituto de Biomedicina de Sevilla, Hospital Universitario Virgen del Rocío/CSIC/ Universidad de Sevilla, 41013 Sevilla, Spain

10 Department of Biochemistry and Molecular Biology, Institut de Neurociències, Faculty of Medicine, M2, Universitat Autònoma de Barcelona (UAB), Bellaterra Campus, Cerdanyola del Vallés, Barcelona, Spain

11 Department of Molecular and Translational Medicine, University of Brescia, Brescia, Spain

12 Faculty of Medicine, University of Vic-Central University of Catalonia (UVic-UCC), Can Baumann, 08500 Vic, Spain 
accumulation, participates in neurite arborization and modulates autophagy. Thus, we determined for the first time that RAC1/ ced-10 participates in Parkinson's disease associated pathogenesis and established RAC1/ced-10 as a new candidate for further investigation of Parkinson's disease associated mechanisms, mainly focused on dopaminergic function and survival against $\alpha$ synuclein-induced toxicity.

Keywords Parkinson's disease $\cdot$ Dopaminergic neurons $\cdot$ Alpha-synuclein accumulation $\cdot$ Autophagy impairment $\cdot R A C 1 / c e d-10$

\section{Introduction}

Parkinson's disease (PD) is the second most frequent neurodegenerative disorder in the elderly. While most of cases are sporadic, monogenic PD caused by pathogenic point mutations in PD-associated genes occurs in less than $10 \%$ of cases (reviewed in [1]). The common neuropathological hallmarks of PD include a selective loss of the dopaminergic neurons (DAn) in the Substantia Nigra pars compacta and aggregation of the protein alpha-synuclein $(\alpha-S Y N)$ in the surviving DAn and in the so called Lewy bodies (LB) and Lewy neurites (LN) which are found in the few surviving DAn (reviewed in [2]). $\alpha$-SYN is intrinsically misfolded in pathological conditions such as PD [3] and forms multiple conformations, including amyloidogenic oligomers $[4,5]$ implicated in $\alpha$-SYN toxicity [6].

There exist evidences of an essential role of actin cytoskeleton disruptions in both DAn cell death $[7,8]$ and $\alpha$-SYN accumulation [9]. In fact, the cytoskeleton is an important target of $\alpha$-SYN [10] and neuronal microtubule-kinesin function could be impaired by $\alpha$-SYN oligomers [11]. Actin cytoskeletal organization is regulated by small GTPases of the Rho family encompassing Rho, Cdc42 and Rac subfamily members [7]. These proteins act as molecular switches as they alternate between the active GTP-bound and the inactive GDP-bound forms $[8,16]$. GTP binding increases the activity, and the hydrolysis of GTP to GDP renders the protein inactive. More specifically, RAC1 activity is mainly associated with cellular processes involving the regulation of actin polymerization such as cell migration, lamellipodia extension or the phagocytosis of dead cells or engulfment [12]. In addition, RAC1 participates in the extension and retraction of neurites [13] and, together with other members of the Rho family, govern changes in neuronal morphology and the dynamics of neuronal processes (reviewed in [8]).

RAC1 function has been associated with two PD-related genes. We have previously shown in Celegans that RAC1 is ubiquitylated by PARKIN [14], mutated in the juvenile variant of PD. Likewise, Leucine-rich repeat kinase 2 (LRRK2), in which mutations cause the most common form of familial PD [15], strongly and selectively binds to RAC1 [16]. Furthermore, neuronal apoptosis induced in DAn in vitro is correlated with decreased RAC1 activity [17]. In contrast, in a monkey model of PD, it was suggested that aberrant activation of RAC1 in microglia may contribute to enhanced production of ROS underlying the death of neighboring DAn [18]. Therefore understanding the cytoskeletal mechanisms associated with DA cell death and $\alpha$-SYN degradation is important to elucidate other causative agents of the PD pathophysiology.

Autophagic flux is profoundly disrupted in PD patients (reviewed in [1] and $\alpha$-SYN is normally degraded by autophagy [19]. Indeed, autophagy has been associated with PD pathogenesis through several genes, such as LRRK2 [20], $A T G 9 A$ [21] or $A T G 8 / L C 3$ [22], and cellular processes such as lysosomal disruption [23, 24]. In addition, autophagyrelated gene products are required for apoptotic clearance, either in dying cells or through a role in engulfment, in where $R A C 1$ has a pivotal role [25-27].

In the present study we have systematically investigated $R A C 1$ function in three disease models of PD including the following: (a) $C$ elegans models of PD; (b) human-derived neuroblastoma BE(2) (M17) cells stably over-expressing $\alpha$ SYN, wherein amyloidigenic accumulation of $\alpha-S Y N$ is induced by sodium butyrate; and (c) iPSC-derived DAn generated by cell reprogramming of somatic skin cells from patients with monogenic LRRK2-associated PD [20]. Using these models, we determine for the first time that $R A C 1 / c e d-10$ participates specifically in PD-associated pathogenesis and establish $R A C 1 / c e d-10$ as a new candidate to be considered for the investigation of PD-associated mechanisms, mainly focused on DA function and survival against $\alpha$-SYN-induced toxicity.

\section{Results \\ RAC1/ced-10 Cell-Autonomous Depletion in DAn Hampers Dopamine- Associated Behavior in the Presence of a-SYN and Accelerates a-SYN Induced DAn Death in C elegans}

We first investigated the role of $R A C 1 /$ ced-10 in DAn function, by performing behavioral assays through analyzing the DA behavior in ced-10(n3246) mutant animals. The mutation ced10(n3246) is a G-to-A transition resulting in a change of glycine 60 of CED-10 to arginine (G60R) which results in non-null altered function $[28,29]$. The severity of this allele is stronger in contrast to other ced-10 alleles (11). To explore the role of ced-10 in PD pathogenesis, all the experiments included in this study involving the ced-10 gene were performed in a ced- 


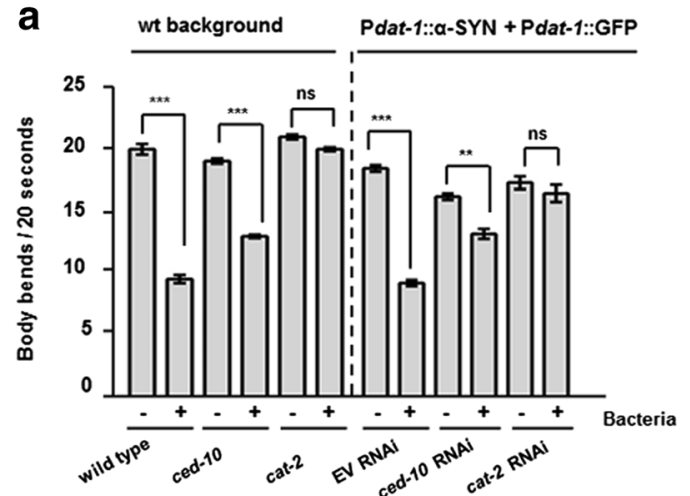

Fig. 1 DAn function is hampered by specific depletion of RACl/ced-10 in DAn expressing $\alpha$-SYN in $C$ elegans, a Modulation of the locomotor rate. Well-fed animals were transferred to assay plates without or with a bacterial lawn $(-/+)$ and $5 \mathrm{~min}$ later, the locomotor rate (body bends every $20 \mathrm{~s})$ of each animal was analyzed. Statistical significance shows comparison of the bending within same genotype animals (wild type, ced-10(n3246) and cat-2 (e1112)) without (-) or with (+) bacteria. RNAi experiments indicate Pdat-1:: $\alpha-\mathrm{SYN}+\mathrm{P}$ dat-1:: GFP worms fed with EV (empty vector) or with the indicated RNAi clones. b Test of ethanol avoidance. The percentage (\%) of ethanol avoidance was

10(n3246) mutant background. To simplify, ced-10(n3246) is named ced-10 from here on.

The basal slowing response is a DA dependent behavior widely used in $C$ elegans for analyzing the functionality of the DA system [30-32]. Briefly, worms decrease locomotion speed when in physical contact with a food source whereas the turn frequency increases when worms leave the food source $[31,33]$. The cat-2 gene encodes the enzyme tyrosine hydroxylase required for the synthesis of dopamine. Accordingly, cat2(e1112) mutant worms have decreased levels of dopamine and altered DA behavior [30,33] and were used as positive control.

The locomotion speed of the nematodes, represented by the body bends every $20 \mathrm{~s}$, was measured in the absence/presence of bacteria (+/-) (Fig. 1a). In a wild type background (wt background), both wild type and ced-10 animals decreased the locomotion speed in the presence of food, thus showing unaltered basal slowing response. In contrast, in cat-2(e1112) mutants the locomotion speed was not significantly decreased by the presence of food (Fig. 1a wt background, and Table 1). Similarly to

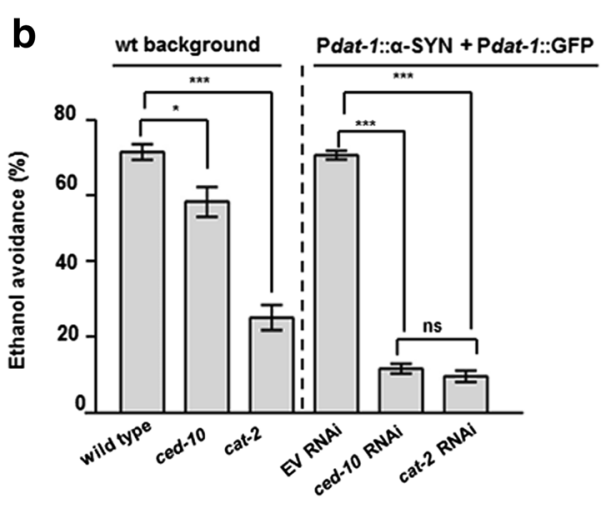

analyzed at the indicated genotypes. Statistical analysis shows comparisons between wild type animals and mutants, and Pdat-1:: $\alpha$ SYN + Pdat-1:: GFP worms fed either with EV (empty vector) or with the indicated RNAi clones. cat-2 (e1112) mutant worms were included as positive controls for both assays. Slow response assay and ethanol avoidance behaviors were hampered in ced-10 depleted animals and not in ced-10(n3246) mutants. Data are mean \pm SEM. $* P<0.05$, $* * P<0.01$, $* * * P<0.001, n s$ non-significant. Statistics: one-way ANOVA, Tukey post hoc test for multiple comparisons. Between 20 and 30 worms were used in three independent replicates

the slow basal response, avoidance against ethanol is a sensory behavior associated with DA signaling [34]. A slight decrease was observed in the ethanol avoidance test performed in ced-10 mutant animals in a wt background (Fig. 1b wt background and Table 2).

The absence of significant dopaminergic behavioral alterations observed in ced-10 mutants might be the consequence of compensating mechanisms existing in the worm that mask the effect of ced-10 function specifically in DAn. To discard any effect of ced-10 function onto DA responses, ced-10 was depleted conditionally in DAn by RNAi, in a C. elegans PD model in which DAn undergo age-dependent neurodegeneration following human $\alpha$-SYN overexpression [35]. In this model, animals express both $\alpha$-SYN and GFP in DAn. Importantly, this is a neuronal-sensitive RNAi strain whereby the impact of RNAi knockdown targeting gene candidates can be selectively examined exclusively in the DAn [36] To simplify, this strain is called Pdat-1:: $\alpha$-SYN + Pdat-1::GFP herein. Animals exposed to ced-10 RNAi showed a mild but significant altered slow
Table 1 Slow dose response quantifications

\begin{tabular}{llcl}
\hline Genotype & $\begin{array}{l}\text { W/o food (body } \\
\text { bends/20 s) }\end{array}$ & $\begin{array}{l}\text { With food (body } \\
\text { bends/20 s })\end{array}$ & $\begin{array}{l}\text { Significance } \\
(P \text { value })\end{array}$ \\
\hline Wild type & $20.00 \pm 0.423$ & $9.55 \pm 0.328$ & $P<0.001$ \\
ced-10(n3246) & $19.05 \pm 0.211$ & $13.00 \pm 0.145$ & $P<0.001$ \\
cat-2 (e1112) & $21.00 \pm 0.191$ & $20.00 \pm 0.162$ & $\mathrm{~ns}$ \\
UA196 on EV & $18.40 \pm 0.255$ & $9.20 \pm 0.257$ & $P<0.001$ \\
UA196 on ced-10 RNAi & $16.00 \pm 0.254$ & $13.15 \pm 0.392$ & $P<0.001$ \\
UA196 on cat-2 RNAi & $17.25 \pm 0.502$ & $16.40 \pm 0.689$ & $\mathrm{~ns}$ \\
\hline
\end{tabular}

Mean and SEM reported, $n=20$. One-way ANOVA 
Table 2 Ethanol avoidance quantifications

\begin{tabular}{lcrl}
\hline Genotype & Avoidance (\% of worms) & $N \quad \begin{array}{l}\text { Significance } \\
(P \text { value })\end{array}$ \\
\hline Wild type & $71.21 \pm 2.120$ & 155 & - \\
ced-10(n3246) & $57.80 \pm 3.957$ & 94 & $P<0.05$ \\
cat-2 $($ el112) & $26.67 \pm 3.335$ & 80 & $P<0.001$ \\
UA196 on EV & $70.35 \pm 1.213$ & $122-$ \\
UA196 on ced-10 RNAi & $12.95 \pm 1.328$ & $95 \quad P<0.001$ \\
UA196 on cat-2 RNAi & $10.902 \pm 1.524$ & $175 \quad P<0.001$ \\
\hline
\end{tabular}

Mean and SEM reported. One-way ANOVA

response assay (Fig. 1a and Table 1). In addition, selective depletion of ced-10 in DAn resulted in similar avoidance against ethanol as cat-2 depleted animals in the ethanol avoidance test (Fig. $1 \mathrm{~b}$ and Table 2). Therefore, in the presence of $\alpha$ SYN, ced-10 function is specifically necessary in $C$ elegans DAn to execute a correct DA behavioral response.

This negative impact of ced-10 depletion on DAn behavior brought us to explore the relevance of ced-10 deficiency in DAn cell death in the above mentioned strain Pdat-1:: $\alpha$-SYN + Pdat-1::GFP. There exist four pairs of DAn in $C$ elegans hermaphrodites, three of them (CEPD, CEPV and ADE) located in the anterior part, and one pair, the PDE, located in the posterior part of the nematode [37]. In this nematode, when human $\alpha$-SYN is expressed in DAn, the six DAn within the anterior region of the worm display progressive degenerative characteristics [38]. To draw parallels between human PD evidenced in aged populations and this worm model, we sought to determine the relevance of ced-10 depletion at 9 days $(\mathrm{L} 4+7)$ post hatching. Cell bodies and neuronal processes were assessed to determine whether these structures were normal or displayed degenerative changes, and consequently considered wild type neurons (Fig. 2). After feeding worms with EV RNAi, $24.93 \pm 2.54 \%$ of animals showed the six anterior wild type DAn. In contrast, ced-10 RNAi knockdown significantly enhanced DA neurodegeneration, $8.33 \pm 1.67 \%$ of animals showed the intact set of DAn $(* * * P<0.001))$ in comparison with EV control (Fig. 2a, b). Animals expressing the fusion protein, CFP::CED-10, under ced-10 promoter, rescued $\alpha$-SYN induced-neurodegeneration $(* * * P<0.001)$ (Fig. 2a, b). We found that neurodegeneration was accelerated already at day 3 and 5 (L4 +1 and L $4+3$, respectively) post hatching (Online Resource 1) thus corroborating the impact of ced-10 in $\alpha$-SYN-induced DA cell death at younger ages.

\section{CED-10 Expression Decreases a-SYN Inclusions Formation in C elegans}

The term phagocytosis refers also to the mechanism by which certain cells engulf and digest other cells and also larger particles or even anomalous inclusions or aggregates [39, 40].
RACl/ced-10 is the converging gene of the engulfment machinery mobilizing actin pseudopodia in phagocytic cells [12]. Therefore, we considered the possibility of ced-10 playing a role in the clearance/phagocytosis of $\alpha$-SYN inclusions. We used a nematode model of PD, in which human $\alpha$-SYN is fused to the yellow fluorescent protein (YFP) under control of the body wall muscle unc-54 promoter, transgene pkIs2386 [Punc-54:: $\alpha-S Y N:: Y F P]$ [41]. With this approach we examined changes in apparent aggregate density or aggregate count of pathogenic $\alpha$-SYN conjugated to fluorescent YFP in muscle cells [42], without considering neuronal side effects. Accordingly, ced-10 animals were crossed with pkIs2386 worms and the number of $\alpha$-SYN aggregates was evaluated in aged worms at 7 days post hatching. This ced-10 mutation increased to 1.5 units the apparent density of $\alpha$-SYN inclusions in comparison to control worms $(0.9 \pm 0.06$ vs. 1.49 \pm 0.06 , respectively; $* * * P<0.001$ ) (Fig. $3 \mathrm{a}, \mathrm{b}$ ) thus suggesting a deleterious effect of the ced-10 mutation in the generation of $\alpha$-SYN aggregates. Importantly, the increase in $\alpha$-SYN apparent aggregates was abolished in transgenic ced-10 mutants expressing the CFP::CED-10 fusion protein (array baEx167[Pced-10::CFP::ced-10]) (0.42 \pm 0.03 in worms expressing CED-10 wild type vs. $1.49 \pm 0.06$ in ced-10 mutant worms respectively and Fig. 3a, b, $* * * P<0.001$ ), showing that the lack of ced-10 is contributing to $\alpha-\mathrm{SYN}$ accumulation.

The number of body thrashes or thrashing have been used extensively to identify modifiers of protein aggregation [41, 43]. Thrashing in $C$ elegans can be measured in liquid media by counting the number of body bends per unit of time [44]. Using this method, we confirmed the observed damaged motility (Fig. 3c and Online Resources 2-4) of the PD worms in a ced-10 background. Whereas a decrease of $27 \%$ in the bending are observed in animals expressing YFP:: $\alpha$-SYN in comparison to the wild type $\mathrm{N} 2$ wild type strain ( $55.01 \pm 7.5 \mathrm{vs}$. $75.96 \pm 2.8$ bends/min, respectively), the number of bends decreases almost $90 \%$ in animals harboring the ced-10 mutation in a YFP::SYN background in comparison with $\mathrm{N} 2$ wild type animals ( $7.93 \pm 4.4$ vs. $75.96 \pm 2.8$ bends/min, respectively) and $70 \%$ in comparison with worms expressing YFP::SYN, without the ced-10 mutation ( $7.93 \pm 4.4$ vs. $55.01 \pm 7.5$ bends/ $\mathrm{min}$, respectively). Thus, increased $\alpha$-SYN in muscle with its concomitant locomotion decrease in ced-10 mutants, indicate the involvement of RAC1/ced-10 in the process of $\alpha$-SYN accumulation in C. elegans.

$\alpha$-SYN variants that form oligomers and protofibrils are associated to the most severe DAn nigral loss in PD models $[1,6]$. To identify the biochemical nature of the apparent $\alpha$ SYN aggregates increased by this ced-10 mutation, worm lysates from pkIs 2386 worms without and with the ced-10 mutation at L4+ 5 days of development, were sequentially extracted by detergent-containing buffers [45] and the amount of $\alpha$-SYN extracted in each fraction was assessed by immunoblotting (Fig. 3d). A faint band of $19 \mathrm{kDa}$ was detected in 


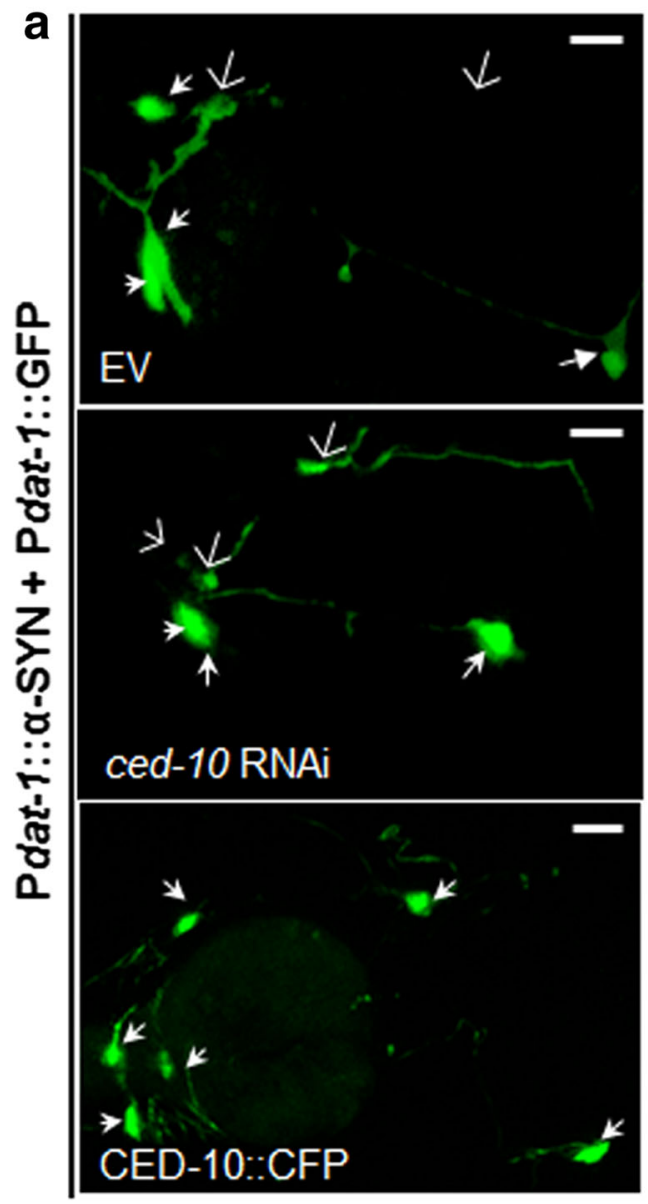

Fig. 2 CED-10 protects DAn from $\alpha$-SYN-induced DA cell death in $C$ elegans. Representative RNAi empty vector (EV) fed worms expressing GFP and $\alpha$-SYN specifically in DAn (Pdat-1:: $\alpha$-SYN + Pdat-1:: GFP) at L4 + 7days ( 9 days post hatching) and fed with empty vector (EV) or ced10 RNAi clones. Filled white arrowhead labels healthy neurons whereas degenerated or missing neurons are labeled with an open arrow. a ced-10 depletion reduces the amount of DAn per worm and the expression of the CED-10::CFP transgene $\{$ baEx167 [Pced-10::CFP:::ced-10] $\}$ delays the DA cell death at the same age. Magnification bar is $30 \mu \mathrm{m}$. b Percentage of Pdat-1:: $\alpha$-SYN + Pdat-1:: GFP worms non-depleted and ced-10

the Tris- $\mathrm{HCl}$ fraction, most probably corresponding to the $\alpha$ SYN monomer staining. The number of oligomeric species is increased in ced-10 mutant animals within all analyzed fractions.

\section{Autophagy Is Impaired in ced-10(n3246) Mutant Animals}

Autophagy is considered one of the main pathways involved in $\alpha$-SYN clearance $[19,46]$. Given the role of RACl/ced-10 detected in $\alpha$-SYN levels and in $\alpha$-SYNinduced DAn cell death, we further sought to determine the participation of ced-10 in the modulation of autophagy in $C$ elegans. To this end, we first crossed ced-10 mutant animals with those carrying the array adIs 2122 b

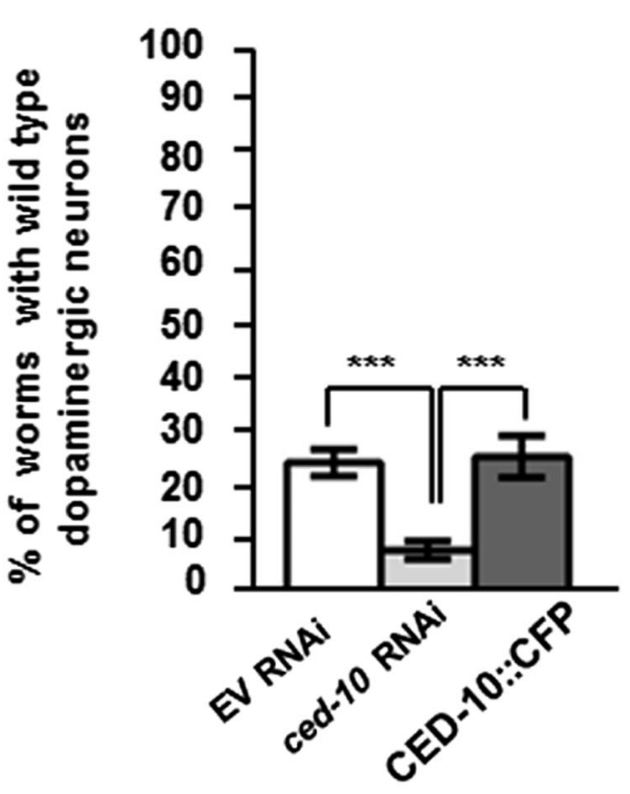

depleted by RNAi, that had the full complement of six anterior DAn at day 7 post L4. The transgenic derivative strain UA281 expressing CFP::CED-10 (CED-10 wild type), carrying the array baEx167 [Pced$10:: \mathrm{CFP}:$ :ced-10] ameliorates the DA cell death. Data are mean $\pm \mathrm{SEM}$. Statistics were obtained by comparing ced-10 RNAi depleted worms or worms containing the CFP::CED-10 array with the corresponding EV fed animals. Statistics: $* * * P<0.001$, one-way ANOVA, Tukey's post hoc test. Number of animals is $30-35$ per condition, and the experiment was repeated three times independently

[Plgg-1::gfp::lgg-1]. The gene lgg-1 encodes a ubiquitinlike protein belonging to the $\mathrm{Atg} 8 / \mathrm{LC} 3$ protein family, and the respective GFP::LGG-1 translational fusion thus allows to monitor autophagosome formation via fluorescence microscopy [47]. To explore the role of ced-10 in autophagy, we manually counted the number of GFP::LGG-1 puncta present in the seam cells [48]. At the L3 stage, the number of puncta present in the seam cells were increased in animals harboring the ced-10 mutation, in comparison to animals without the mutation, in where the GFP::LGG-1 pattern is mainly diffuse (Fig. 4a, b). An increase in the number of GFP::LGG-1 puncta may result from either elevated or impaired autophagic flux $[48,49]$. Therefore, we investigated the involvement of RACl/ced-10 in autophagic pathways 
a
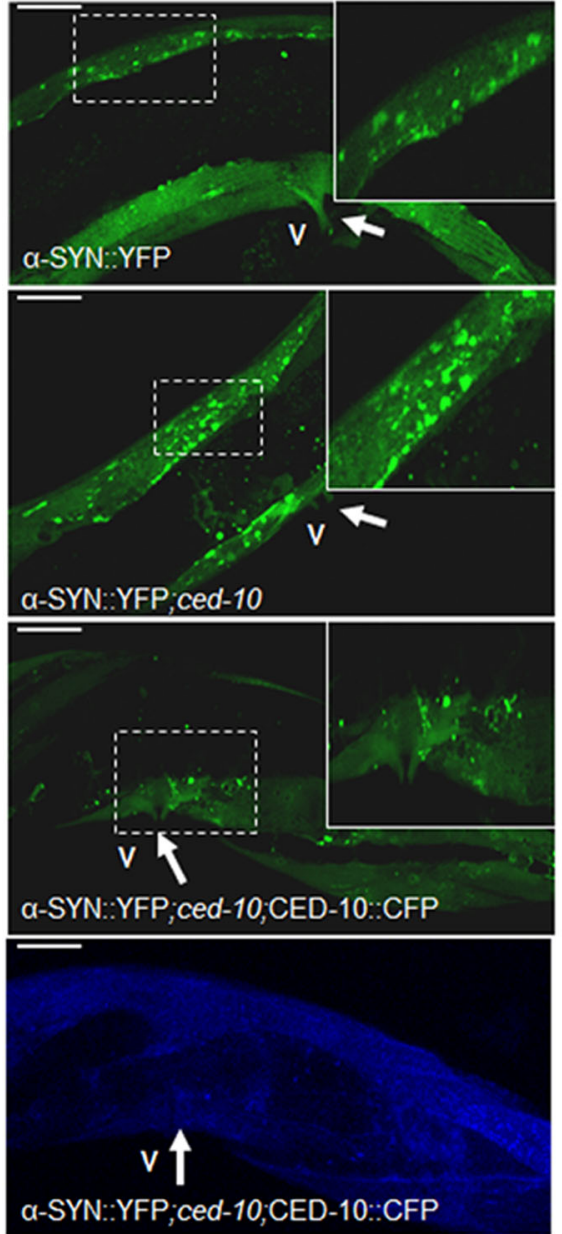

Fig. 3 ced-10 decreases $\alpha$-SYN inclusions in $C$ elegans. a Representative confocal pictures obtained from animals containing the genomic array pkIs2386 [Punc-54.: $\alpha$-SYN.:YFP] expressing $\alpha-\mathrm{SYN}$ in body wall muscle cells at L4 +5 days of development ( 7 days post hatching). Green staining in all figures represents $\alpha$-SYN::YFP inclusions in muscle cells. The vulva (V, thick arrow) was used as a reference to analyze the same central section in all worms. A representative area was highlighted and expanded in each panel, to better visualize the $\alpha$-SYN::YFP accumulation. a (first panel) $\alpha$-SYN inclusions were detected in a $C$. elegans model of $\alpha$-SYN miss folding in which $\alpha-\mathrm{SYN}$ is expressed under the control of the unc-54 promoter. a (second panel) $\alpha$-SYN apparent aggregates are increased in ced10(n3246) mutant nematodes. a (third panel) CFP::CED-10 expression (array baEx167 [Pced-10::CFP::CED-10]) decreased the number of $\alpha$ SYN inclusions in a ced-10(n3246) background. a (fourth panel) The blue fluorescence marker (CFP) represented the endogenous expression

by analyzing the impact of the ced-10 mutation in the autophagy-associated reporter strain bpIs51 [Psqst-1:sqst-1::gfp + unc-76(+)]. The C. elegans SeQueSTosome-related protein, SQST-1, exhibits sequence similarity to mammalian SQSTM1/p62 and is degraded by autophagy [48, 50]. As such, autophagy impairment is often associated with SQST-1::GFP accumulation [48-50]. Similarly to the results obtained with
C

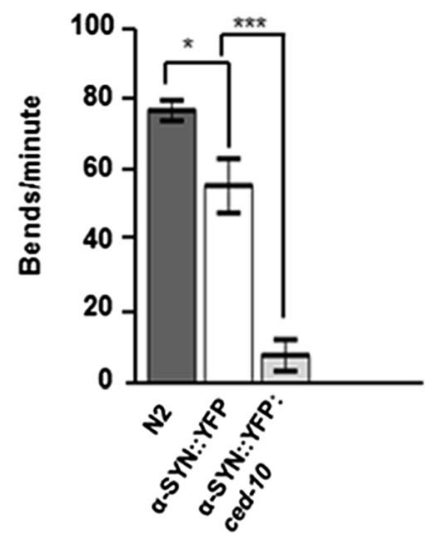

of CED-10 in a ced-10(n3246) background for rescuing $\alpha$-SYN accumulation. Magnification bar is $10 \mu \mathrm{m}$. b Quantification of the number of $\alpha$-SYN inclusions per area. Data are mean \pm SEM. Between 30 and 35 animals were analyzed per genotype. Three different transgenic lines expressing CFP::CED-10 were generated and analyzed independently. Statistics: one-way ANOVA with a Tukey post hoc test. $* * * P<0.005$. $\mathbf{c}$ The movement of YFP:: $\alpha$-SYN animals is hampered by the mutation ced-10n(3246). Thrashing behavior (bends/min) was video recorded and the resulting images were analyzed by the ImageJ software. Data are mean \pm SEM. Between 20 and 30 animals were recorded per experiment and the same experiment was repeated 3 independent times. $d$ Immunoblotting analysis of protein extracts from 5 days post L4 old YFP:: $\alpha$-SYN synchronized animals, using anti- $\alpha$ - SYN antibody without and with the ced-10(n3246) mutation (wild type and ced-10 respectively). The amount of $\alpha-\mathrm{SYN}$ insoluble species was increased by the ced-10(n3246) mutation.

the GFP::LGG-1 reporter, ced-10 mutant animals displayed increased SQST-1::GFP internal density (Fig. $4 \mathrm{c}-\mathrm{e})$. Whereas SQST-1::GFP staining was barely detected in wild type animals (Fig. 4c, d upper panels, and e), ced-10 worms displayed SQST-1::GFP accumulation (Fig. 4c, d bottom panels, and e). Cumulatively, these results suggest a role of $R A C 1 / c e d-10$ in the regulation of autophagy. 


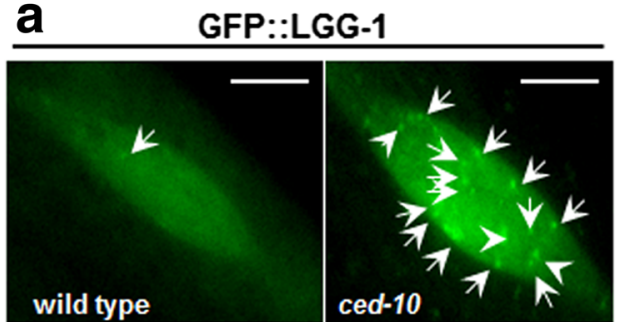

b

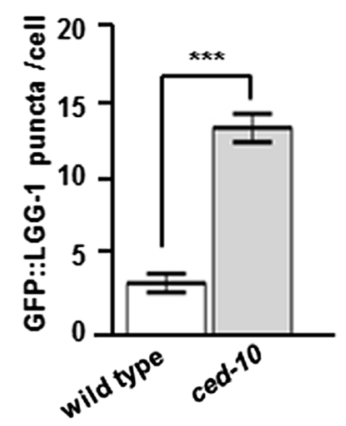

Fig. 4 Autophagic markers accumulate in ced-10 mutant worms. a L4 worms expressing the reporter GFP::LGG-1 (adIs2122 [Plgg1::GFP::lgg-1; rol-6(su1006)] in hypodermal seam cells, without (left panel, wild type) and with (right panel) the ced-10(n3246) mutation. GFP::LGG-1 puncta are labeled with an filled arrow. Magnification bar is $5 \mu \mathrm{m}$. b The bar graph indicates the number of GFP::LGG-1 foci per cell at the indicated genotypes. These results are mean \pm SEM of three independent experiments performed in triplicate. Statistics is Student's $t$ test. $* * * P \leq 0.001$. c, d Worms expressing the autophagy reporter SQST1::GFP (bpIs151[Psqst-1::sqst-1::GFP]) were crossed with ced-10(3246) animals and the GFP fluorescence intensity (FI) was analyzed under a

\section{Human RAC1 Expression Reduces a-SYN Accumulation and Amyloidogenic Aggregation in a Neuroblastoma Cell Line}

We further explored the effect of $R A C 1$ on $\alpha$-SYN accumulation using a stable BE(2)-M17 neuroblastoma cell line over-expressing wild type $\alpha$-SYN. As previously reported [51], differentiation with retinoic acid (RA) and treatment with the histone deacetylases inhibitor sodium butyrate (SB) increased $\alpha$-SYN expression by twofold and induced the accumulation of small $\alpha$-SYN cytoplasmic aggregates (Online Resource 5a-b). Differentiated cells treated with $\mathrm{SB}$ were transduced with a lentiviral vector (LV) expressing either RAC1 wild type (WT)GFP (in which RAC1-GTP and RAC1-GDP coexist) or RAC1 constitutively active (CA)-GFP (only expressing RAC1-GTP) and analyzed 4 days post-transduction, using the empty vector (Control-GFP) as a control. Infection with both, RAC1 (WT) and RAC 1(CA) decreased $\alpha$ SYN expression level (Online Resource 5b) and
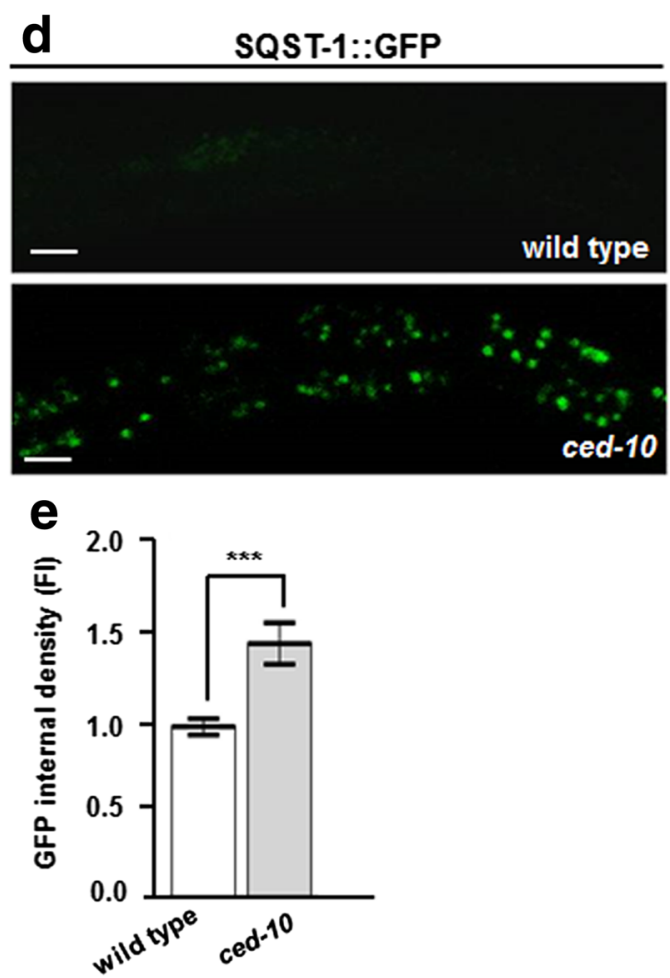

fluorescence (c) or a confocal (d) microscope. d (upper panel) L4 animals expressing the array SQST-1::GFP without induction of the GFP reporter in normal conditions. d (bottom panel) The ced-10(n3246) mutation increased GFP intensity and aggregation. Magnification bar is $20 \mu \mathrm{m}$. e Normalized fluorescence intensity (FI) observed in SQST-1::GFP animals without and with the ced-10(n3246) mutation. Thirty animals were analyzed per genotype. Data are represented as the mean \pm SEM and were obtained by comparing wild type animals (without the ced10 (n3246) mutation) with ced-10 mutated animals. Statistics, $* * * P<0.001$, Student's $t$ test

aggregation, as shown by Thioflavin $\mathrm{S}$ (ThyoS) dye (Fig. 5), which specifically stains cross-beta sheet fibrils, such those forming amyloid aggregates [52]. The area covered by ThyoS -positive $\alpha$-SYN aggregates per cell was decreased by $90 \%$ in $\mathrm{RAC} 1$ (WT) and RAC1(CA) infected cultures (Fig. 5) thus suggesting a role of RAC1 in either the formation or clearance of toxic $\alpha$ SYN species and corroborating the data obtained by western blot in the nematode.

\section{High RAC1 Activity Reduces a-SYN Levels and Increases Neurite Arborization in PD Patient-Specific Midbrain iPSC-Derived DAn}

Lastly, to connect the nematode data with the human PD, we differentiated DAn upon cell reprogramming of skin fibroblasts into induced pluripotent stem cells (iPSC) from PD patients carrying the G2019S (G/S) mutation in the LRRK2 gene. While preserving the patient genetic background, this model exhibits some characteristic 

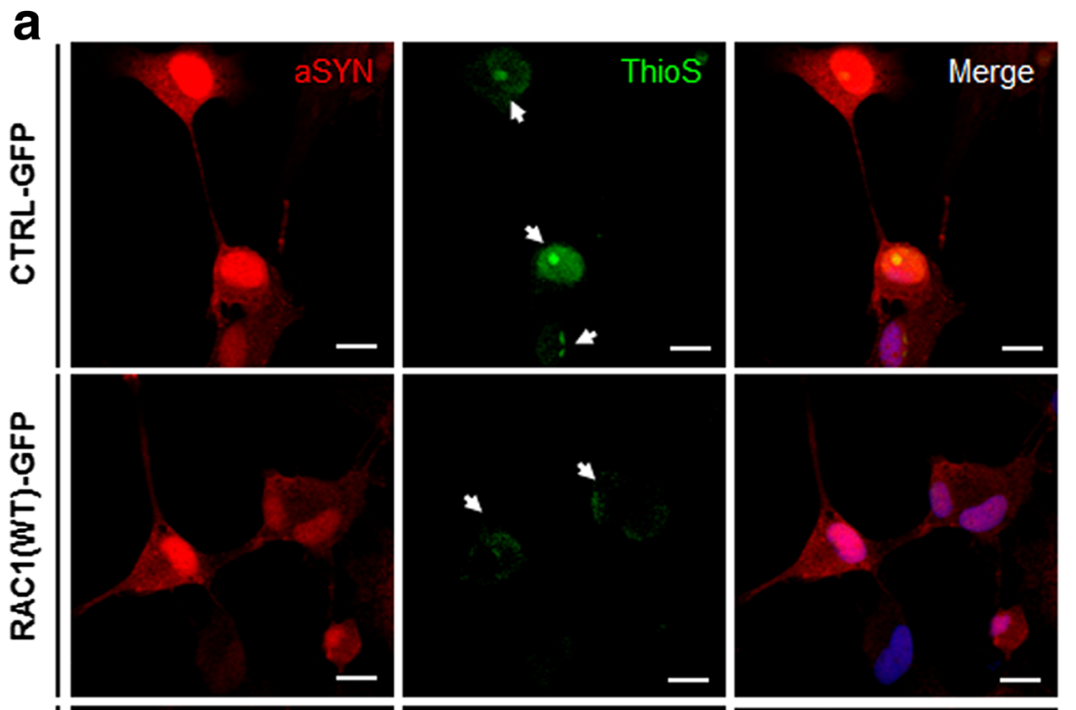

\section{b}
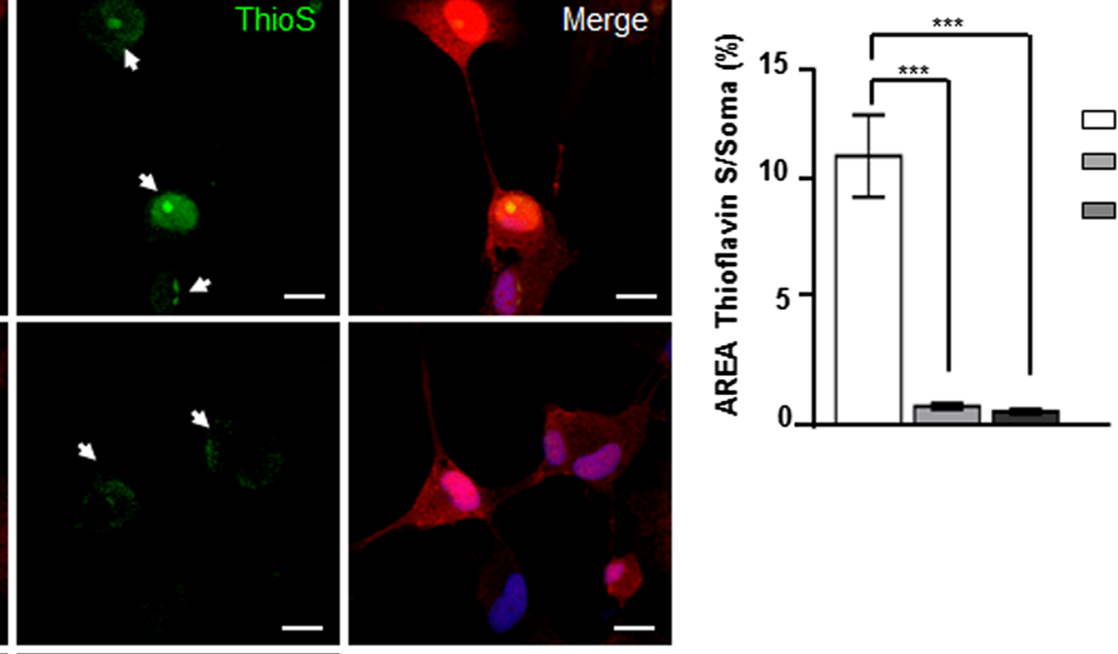

CTRL-GFP

RAC1(WT)-GFP

RAC1(CA)-GFP
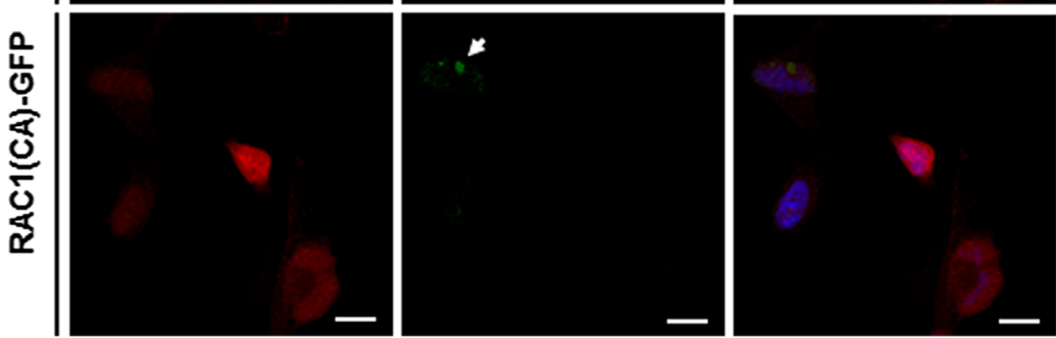

Fig. 5 Rac 1 activity decreases $\alpha$-SYN accumulation and aggregation in the neuroblastoma cell line BE(2)-M17. a Representative confocal images of $\alpha$-SYN over-expressing cells induced with $10 \mu \mathrm{M}$ retinoic acid (RA) and treated with $10 \mathrm{mM}$ sodium butyrate (SB) for $36 \mathrm{~h}$. Cells were transduced with ControlGFP (upper row), RAC1 (WT)-GFP (middle row) and RAC1 (CA)GFP (bottom row), and co-stained for Thioflavin S (green) and $\alpha$ SYN (red). Arrows indicate Thioflavin $\mathrm{S}$ positive aggregates with

amyloidal structure. b Bar graph showing the quantitative analyses of the neuronal soma area (in percentage \%) covered by Thioflavin $\mathrm{S}$ positive stain in individual cells transduced with (WT)- or (CA) RAC1 or with the corresponding control. $N=14$ (EV), $N=25$ (WT), and $N=24$ (CA), from at least three independent experiments. Data are presented as mean \pm SEM. Statistics, $* * * P<0.001$, one-way ANOVA with Bartlett's test correction followed by post hoc Tukey test. Scale bars represent $10 \mu \mathrm{m}$

features and cellular phenotypes of PD including reduced axonal outgrowth, $\alpha$-SYN accumulation, $\alpha$-SYN-induced DAn cell death, and impaired autophagy [20]. Therefore, it represents a suitable tool to contextualize and compare the nematode encountered data.

At 30 days of differentiation, patient iPSC-derived DAn do not show overt morphological signs of neurodegeneration [20]. However, almost $40 \%$ of DAn positive for the DA marker tyrosine hydroxylase (TH) showed detectable amounts of $\alpha$-SYN [20]. For this reason, and to explore early events affecting PD, we next investigated the contribution of RAC1 in PD by rescuing $\alpha$-SYN accumulation in DAn. For this purpose, PD-iPSC-derived midbrain DAn (at 30 days of differentiation when $\alpha$ SYN accumulation is already evident), were transduced with lentivirus (LV) expressing either RAC1 wild type (RAC1 (WT)-GFP or a highly active form of RAC1 (RAC1 (CA-GFP), and LV-GFP as control (ControlGFP) (Online Resource 6a, c and d), and analyzed 7 days after transduction. We found that LRRK2-PD-derived DAn, transduced with Control-GFP showed significant increase in $\alpha$-SYN content in comparison with non-PDderived DAn (Fig. 6a, first and second panels, and scatter dot plot) confirming previous results [20]. A $18 \%$ decrease in $\alpha$-SYN accumulation was observed in PDderived cells infected with RAC1 (WT)-GFP, and it was even stronger $(48.15 \%)$ in PD-derived cells infected with RAC1 (CA)-GFP (Fig. 6a, third and fourth panels, and scatter dot plot, $* * * P<0.001)$. By analyzing the number and length of neurites, to explore the capacity of Rac1 in rescuing neuronal degeneration (Online Resource 7), we found a decrease in neurite arborization in PD-derived DAn (Fig. 6b), confirming previous reports showing reduction in neurite length/branching and defects of Rac signaling in LRRK2-associated parkinsonism [53]. Importantly, overexpression of RAC1 (CA)-GFP, but not RAC1 (WT)-GFP, was associated with significant increase of neurite arborization (Fig. 6b, fourth panel and left graph), consistent with a role for RAC1 in organizing the actin cytoskeleton [58]. The neurite length was not rescued in any of the conditions tested (Fig. 6b, right graph). 


\section{a}
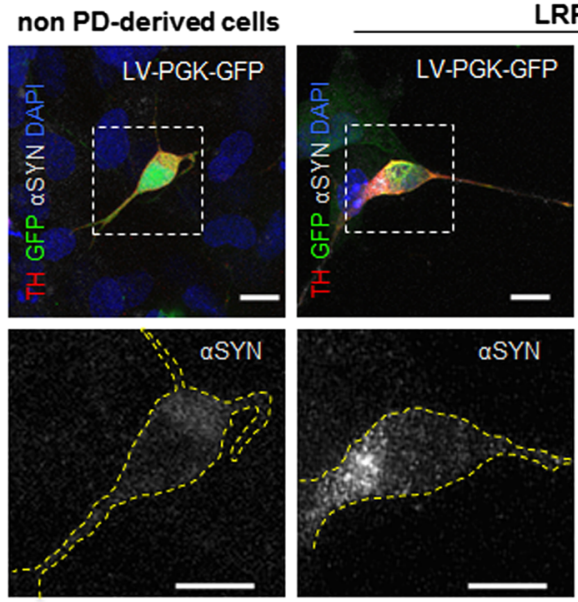

LRRK2-PD-derived cells
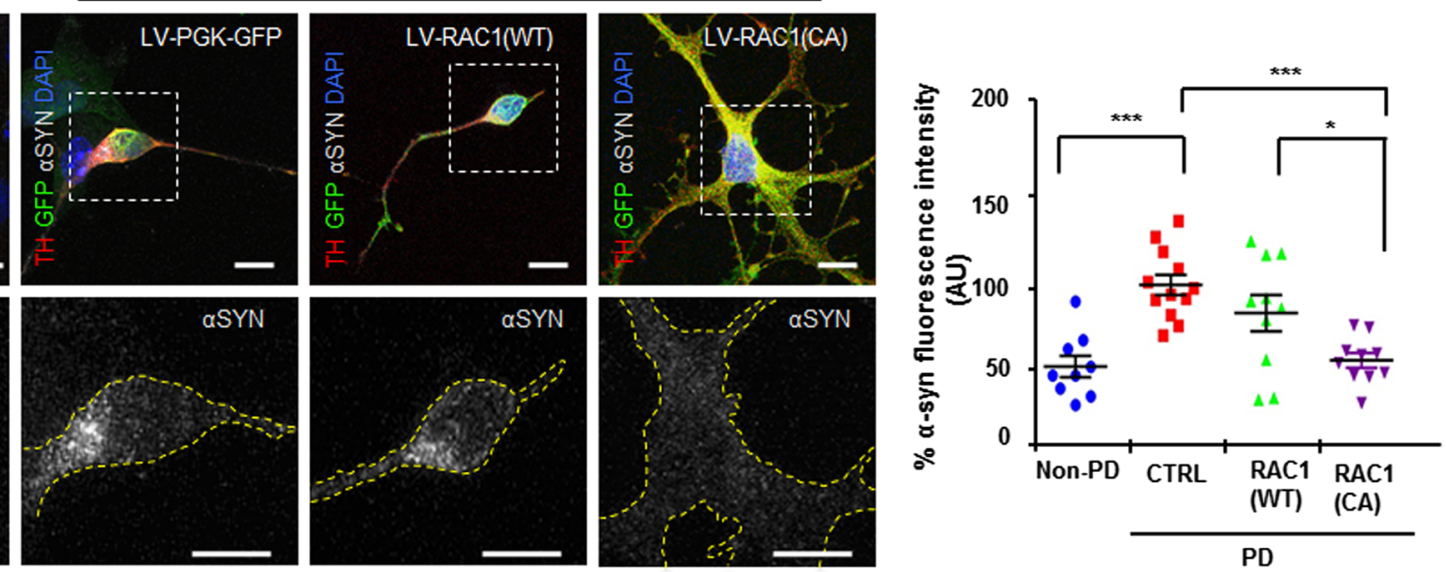

b
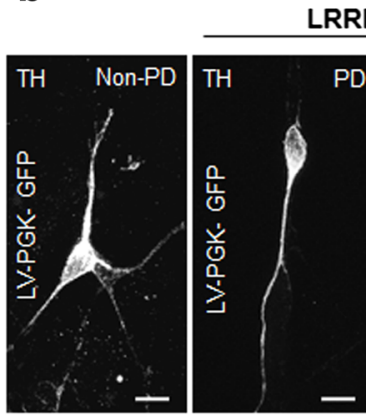

LRRK2-PD-derived cells
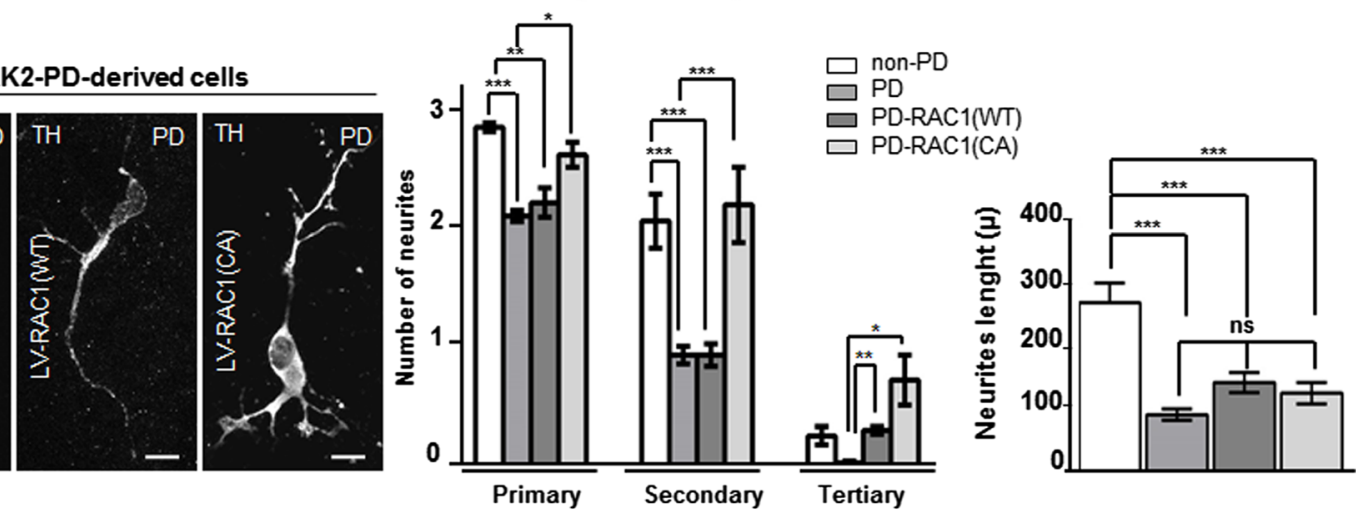

Fig. 6 Rac 1 activity rescues $\alpha$-SYN accumulation and neurite degeneration in early LRRK2-PD-derived DAcells. a First row shows confocal images of non-PD (first panel) and LRRK2-PD-iPSC-derived DAn (second, third, and fourth panels) at 30 days of differentiation transduced with Control-GFP (first and second panels), RAC1 (WT)GFP (third panel) and RAC1 (CA)-GFP (fourth panel), and co-stained for GFP (green), Tyrosine hydroxylase (TH) (red) and $\alpha$-SYN (gray). Nuclei are counterstained with DAPI, shown in blue. Second row shows confocal images representing the expanded pictures of the corresponding above neurons highlighted within the white dashed square, evidencing $\alpha$-SYN staining. Dot plot shows the quantification of the average (in \%) of $\alpha$-SYN fluorescent intensity in every analyzed neuron positive for TH and GFP. Statistical analysis is the result of comparing $\alpha$-SYN staining intensity of non-PD with LV-transduced DAn. Data is the average of at least two-independent experiments and are presented as mean \pm SEM. Statistics is one-way ANOVA with a Tukey's post hoc analysis $* * P<0.01$. b Representative confocal

micrographs of single DAn derived from non-PD (first panel) and LRRK-2PD patients, transfected with Control-GFP (second panel), RAC1 (WT)-GFP (third panel) or RAC1 (CA)-GFP (fourth panel). Extension bars are $10 \mu \mathrm{m}$. (Left graph) This bar graph represents the number of neurites per neuron (primary, secondary and tertiary), according to the indicated transduction, in non-PD and in PD-derived cells. Statistical analysis is the result of comparing neurite number nonPD with LRRK2-PD-derived DA cells transduced with RAC1 (WT)GFP or RAC1 (CA)-GFP. Data is the average of at least twoindependent experiments and are presented as mean $\pm \mathrm{SEM}$. Statistics is One-way ANOVA, $* P<0.05$ and $* * P<0.01$. (Right graph) Quantitative analyses of the neurite length (in $\mu \mathrm{m}$ ) in DAn derived from non-PD and LRRK2-PD-derived patients. Statistical analysis is the result of comparing neurite number non-PD with LRRK2-PD-derived DA cells transduced with RAC1 (WT)-GFP or RAC1 (CA)-GFP. Data are presented as mean \pm SEM. Statistics is one-way ANOVA. $* * * P<0.001$

By transcriptome analysis we have previously reported that iPSC-derived DAn from PD patients exhibited a large number of gene expression changes. More specifically, we identified 437 differentially expressed genes (DEGs) in PD vs. controls of which 254 were up-regulated in PD patients and 183 were down-regulated [54]. Here, to gain further insight into the canonical pathways affected by differential gene expression detected at early PD, we performed a biological enrichment analysis at 30 days of differentiation, by using the software and databases of Ingenuity Pathway Analysis (IPA). We found that the signaling by Rho family GTPases pathway was the

top-1 statistically most significant canonical pathway in PD patients compared to controls $\left(P=2.56 \times 10^{-4}\right)$ (Online Resource 8). From the total of 245 members comprised in this pathway, 16 DEGs of which 9 genes were upregulated and 7 down-regulated in PD DAn (Online Resource 9). Interestingly, the top 2 statistically most significant canonical pathway was the related Rho GDI pathway $(P=7.91 \times$ $\left.10^{-4}\right)$. Overall, the results from this unbiased biological enrichment analysis identifies Rho family GTPases as top deregulated canonical pathway in iPSC-derived DAn from PD patients. 


\section{RAC1 Activity Increases the Long-Term Survival of PD-Patient-Derived DAn and Alleviates the Impairment of Autophagy}

To assess whether the protective effect of RAC1 in reducing $\alpha$-SYN levels correlated with increased survival rates over time, neurons were further cultured for 75 days (Online Resource 6b) by co-culturing them over a monolayer of mouse post-natal cortical astrocytes [55], which supported viable cultures of DAn for up to 75 days [20].

After this time span, differentiated cultures from genetic LRRK2-PD- patient derived DAn showed higher numbers of apoptotic DAn when compared to those derived from healthy subjects [20] and Fig. 7(a, b, and e). Overexpression of both RAC1 (CA)-GFP and RAC1 (WT)-GFP prevented cell death by reducing the amount of DAn positively stained for cleaved caspase-3 to the levels of the non-PD-patient-derived DA cells (Fig. 7(c-d and e)). We and others have described that LRRK2 G2019S mutation has negative effects in the autophagic flux by seemingly impairing autophagosome-lysosome fusion [20]. In this specific case, RAC1 CA (RAC1-(CA) but not RAC1 wild type (RAC1-(WT) displayed autophagosome vesicle numbers similar to those of the non-PD-patient-derived neurons (Fig. 7). Therefore, these results suggest a mechanism by which a better performance of the autophagic clearance promoted by RAC1 alleviates the accumulation of aggregationprone proteins, such as $\alpha$-SYN, thus contributing to increase the survival of DAn.

In conclusion, these results obtained in DAn derived from LRRK2-associated PD patients are in line with findings in the nematode models of PD, where RAC1 activity is directly involved with DAn survival in the presence of $\alpha$-SYN, $\alpha$-SYN inclusions formation and autophagic mechanisms.

\section{Discussion}

Here, we demonstrate in C elegans and in human-derived PD cells that $R A C 1 /$ ced-10 participates in the main pathogenic manifestations of PD such as DAn death, $\alpha$-SYN accumulation and impaired autophagy. Besides, the results obtained in the nematode, suggest a role of ced-10 modulating DA behavior in the presence of $\alpha$-SYN. Furthermore, RACl function is associated with the considered toxic $\alpha$-SYN species. Overall, in this manuscript we propose $R A C 1 /$ ced-10 as a potential therapeutic target for the treatment of PD-related disorders.

\section{RAC1/ced-10 and DAn Death}

Previous research has shown that Rac GTPases play an essential neuroprotective and pro-survival role in neuronal models and diseases [17, 56-58]. Indeed, our in depth analysis of the RAC1 signaling pathway arose from the transcriptomic data in human
Fig. 7 RAC1 activity increases the long-term survival of PD-derived DAn and alleviates autophagy impairment. (A-D) Confocal images of non- $\mathrm{PD}(\mathrm{A})$ and $\mathrm{PD}-\mathrm{PSSC}$-derived DA cultures transduced with ControlGFP (B), RAC1 (WT)-GFP (C), and RAC1 (CA)-GFP (D), stained for GFP (green), TH (red) and cleaved Caspase-3 (white). A representative area was highlighted in dashed lines and expanded in below panels. The corresponding lower insets show higher magnification images of each separate channel, cleaved caspase-3, TH and RAC1-GFP respectively. $\mathrm{TH}+$ neurons, stained with Caspase- 3 and transduced with RAC1-GFP constructs are labeled with white arrow tips. Caspase-3 posoNuclei are counterstained with DAPI, shown in blue. PD-derived cells transduced with Control-GFP (second panel) showed increased numbers of triple positive $\mathrm{TH} / \mathrm{GFP} /$ Caspase 3 staining in $\mathrm{TH}+$ neurons in comparison to the other conditions tested, where cells were non-PD (first panel left) or transduced with RAC1 (WT)-GFP and RAC1 (CA)-GFP (third and fourth panels respectively). Extension bar from A-D is 20 and $10 \mu \mathrm{m}$ for top and bottom panels respectively. e Bar graph showing the quantification of the number of TH/GFP double-positive neurons presenting cleaved Caspase-3 staining. Data is the average of at least two-independent experiments and are presented as mean $\pm \mathrm{SEM}$. Statistics, $* P<0.05$, $* * P<0.01$, and $* * * P<0.001$, Two-way ANOVA, Tukey post hoc test. f Confocal images of non-PD (left panel) and PDiPSC-derived DA cultures transduced with Control-GFP (second row), RAC1 (WT)-GFP (third row), and RAC1 (CA)-GFP (fourth row), stained for GFP (green), TH (red) and LC3 (gray). Non-PD cells (first row) and PD cells transduced with RAC1 (CA)-GFP (fourth row) showed similar amount of LC3-II positive vesicles in TH+/GFP+ neurons. Nuclei are counterstained with DAPI, shown in blue. Extension bar is $5 \mu \mathrm{m}$. $\mathbf{g}$ Bar graph showing the quantification of the neuronal soma area (in percentage $\%$ ) covered by LC3-II positive vesicles in at least 15 DA neurons. Data is the average of at least two-independent experiments and are presented as mean \pm SEM. Statistics, $* P<0.05$, Two-way ANOVA, post hoc Tukey test

iPSC-derived DAn from PD patients, showing altered Rho signaling as top deregulated pathway, points to this same direction (Online Resources 8-9). However, Rac GTPases modulation in different cell types is much more complex. Loss of RAC GTPase activity may contribute to the death of DAn while increased Rac-GTP activity in microglia may contribute to the formation of toxic ROS [59]. Hence, this complicated RAC1 modulation depending upon the tissue and the ROS state, might explain the behavioral differences observed between whole ced10 mutant animals and $R A C l / c e d-10$ specifically depleted in DAn in the presence of $\alpha$-SYN (Fig. 1). Interestingly, a cell non-autonomous function for hypodermal RAC1/ced-10 in the maintenance of axonal survival has been recently proposed in C. elegans [60]. Consequently, the influence of RAC1 activity in the neighboring tissues cannot be obviated.

There exist positive correlation between neuronal apoptosis and decreased RAC1 GTPase activity [17]. Very different cellular models, such as human lymphoma cells or primary cerebellar granule neurons, suggest the inhibition of caspaseinduced apoptosis by RAC1, whereby AKT-dependent prosurvival pathways and the consequent Bcl-2-associated death protein $(\mathrm{BAD})$ phosphorylation were downstream and activated by RAC1 [59, 61].

The activation of the AKT by RAC1, also participates in the cytoskeleton reorganization and cellular growth $[62,63]$ 

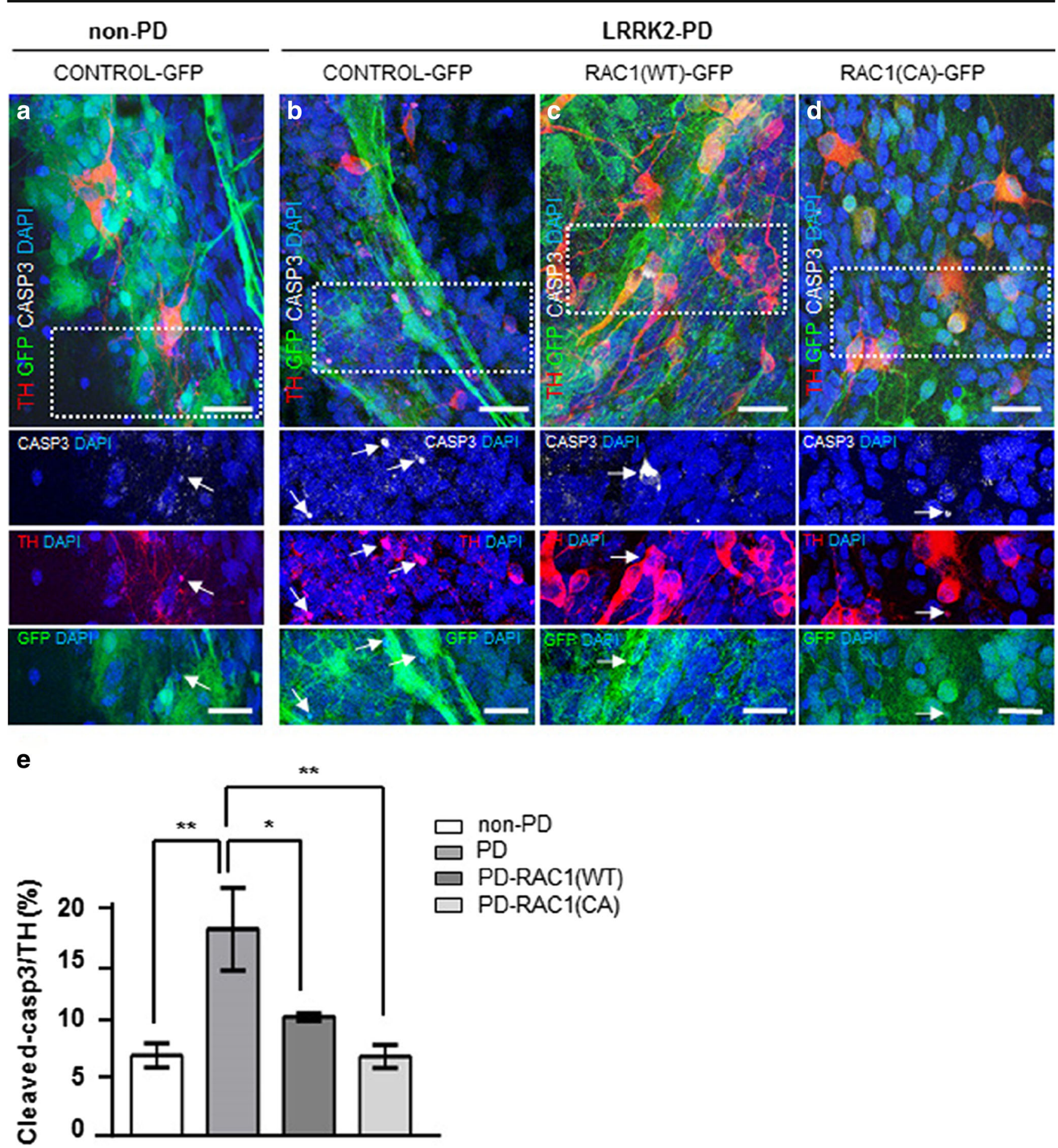

and a failure to maintain the integrity of DAn after they are formed could cause DAn death [64]. Accordingly, RAC1modulated processes involved in the maintenance of cell integrity, might be crucial for cell survival.

LRRK2-PD-patient-derived DAn show increased neurite numbers after being transduced with RAC1 (Fig. 6), thus expanding the role of this GTPase in the maintenance and in the generation of new neurites [13], and thus contributing accordingly to DAn survival (Fig. 7). Therefore, our results are in accordance with LRRK2 inducing neurite retraction through diminished RAC1 GTPase activity [16]. Surprisingly, neurite length was not rescued with any of the infected RAC1 constructs in the present manuscript. Differences between results can be explained based on the different cellular models used in both laboratories, since our results are provided directly from PD-derived cells, whereas the neuroblastoma cell line SHSY5Y is the model used by Chan et al. [16]. In addition, the existing actin-microtubule 


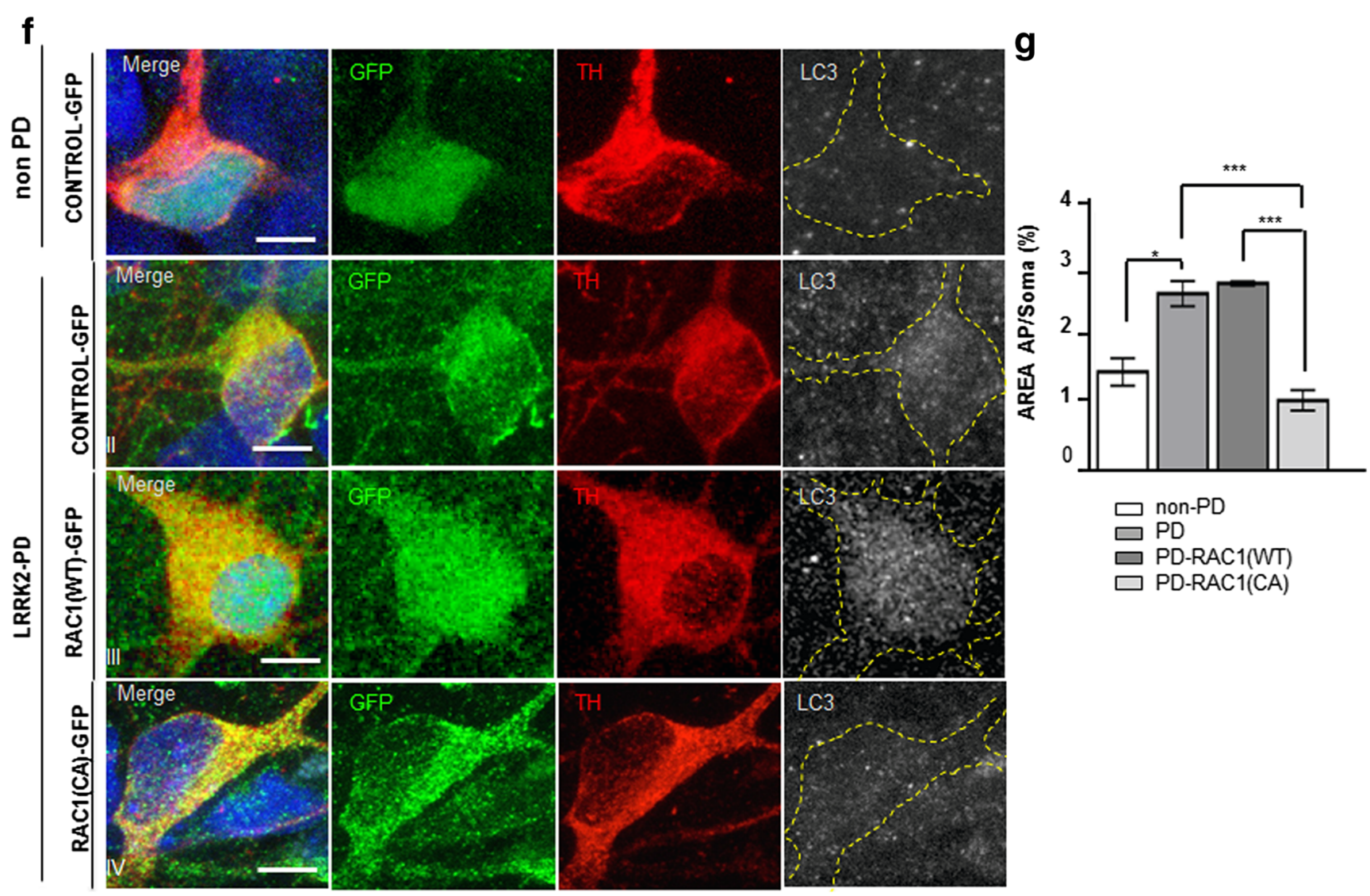

Fig. 7 (continued)

cross talk in the process of neurite outgrowth and elongation has to be considered $[65,66]$. Microtubules are the main cytoskeletal components of neurites [66] and decreased stability of microtubules is a common feature of neurodegenerative diseases [67]. Interestingly, LRRK2-PD variants are characterized by defects in microtubule associated processes [68] and LRRK2 regulates microtubule stability [69].However, extension and navigation of neurites are normally driven by actin-rich growth cones and inhibition of microtubules dynamics does not stop neurite outgrowth [66]. Thus, our results are consistent with cellular dysfunction in PD, with RAC1 modulating actin-associated mechanisms better than in microtubule linked processes.

\section{RAC1/ced-10 and a-SYN Accumulation}

One of the main factors linked with DAn death in PD progression is $\alpha$-SYN accumulation. $\alpha$-SYN overexpression in model systems, and its concomitant aggregation and deposition precede neuronal cell death. In the case of DAn, its degeneration is influenced by intracellular and extracellular $\alpha$-SYN accumulation, mainly in its oligomeric form [70]. Interestingly, extracellular oligomeric $\alpha$-SYN impairs RAC1 activity in neuroblastoma cells [70]. Considering the increased intracellular $\alpha$-SYN aggregates together with increased oligomeric $\alpha$-SYN species observed in ced-10 mutant animals, we hypothesize that altered $R A C 1 /$ ced-10 function might accelerate $\alpha-\mathrm{SYN}$ accumulation and the formation of $\alpha-\mathrm{SYN}$ oligomers which might bind concurrently RACl/ced-10 [70] thus increasing the severity of RACl/ced-10 altered function. Moreover, and considering the modulation of the actin cytoskeletal dynamics by $\alpha$-SYN [71], a synergistic regulation between $\mathrm{RAC} 1$ and $\alpha$-SYN cannot be excluded.

Additional experiments, where the amount of $\alpha$-SYN could be tightly controlled, will provide some clues about the relevance of $\mathrm{RAC} 1 / \alpha$-SYN interaction in the progress of PD.

$\alpha$-SYN accumulation in PD patients is associated with failure of the two major protein breakdown pathways, the ubiquitin proteasome system (UPS) and autophagy [72-74], which, in cooperation, reduce the misfolded protein burden [75]. Stably increased levels of $\alpha$-SYN can lead to impaired proteasome function [76] and ced-10 is proteasome regulated in the phagocytosis of dead cells [14]. Hence, we hypothesize that increased $\alpha$-SYN in a ced-10 mutant background might reinforce the severity of the ced-10 mutation in the degradation of $\alpha$-SYN, due to the interaction of $\alpha$-SYN with the proteasomal machinery. 
Overexpression of $\alpha-\mathrm{SYN}$ results in the inhibition of autophagy [77]. Here we suggest RAC1/ced-10 being necessary for autophagy to occur (Figs. 3 and 7). Accordingly, $\alpha-\mathrm{SYN}$ accumulation, due to ced-10 impairment, might increase the severity of ced-10 mutation. Therefore we add RAC1 to the already proposed feedback loop between proteasome activity and autophagy [1] and we propose that a tight regulation of RAC1 function is required to avoid excess of $\alpha-S Y N$ accumulation and the concomitant cell death.

Impaired autophagy associated with LRRK2 mutations are already reported [78, 79], with the G2019S mutation showing less autophagic activity [80]. In the context of LRRK-2 induced phenotypes, we propose RAC1-LRRK2 interaction as relevant factor favoring autophagy to occur, and helping in the clearance of $\alpha$-SYN aggregates, thus warranting the proper neurite growth and maintenance.

Future scientific research is needed to unravel the mechanisms associated with PD-related disorders for finding efficient therapies. In light of our results the pharmacological modulation of RAC1 and RAC1-derived signaling pathways could be of therapeutic value.

\section{Experimental Procedures}

\section{Worm Experiments}

\section{C. elegans Strains}

Nematodes were maintained using standard procedures [81]. We obtained the strains CB1112 cat-2(e1112), NL5901, unc-

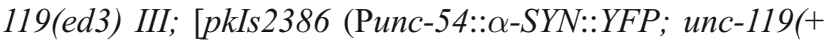
))]; DA2123 adIs2122 [Plgg-1::lgg-1::GFP; rol-6(su1006)] and HZ589, bpIs151 [Psqst-1::sqst-1::GFP; unc-76(+)] IV; him-5(e1490) $V$ from the Caenorhabditis elegans Genetic Center (CGC). The strain BR3579, ced-10(n3246) was a generous gift from Dr. Ralf Baumeister (Albert-Ludwing University, Freiburg/Breisgau, Germany).

The strain BR3579 was crossed with NL5901 animals to generate the strain EDC101, unc-119(ed3) III; pkIs2386 [Punc-54:: $\alpha-S Y N:: Y F P ; ~ u n c-119(+)]$; ced-10(n3246).

The following strains is used to analyze the DAn degeneration as reported in Harrington et al., 2010 [36]: Pdat- $:: \alpha-$ SYN +Pdat-1::GFP from the main text is named UA196, with the genotype: sid-1(pk3321); baIn33 [Pdat-1::sid-1; Pmyo2::mCherry]; baIn11 [Pdat-1:: $\alpha-\mathrm{SYN} ;$ Pdat-1::GFP].

For autophagy experiments, males from the DA2123 strain [Plgg-1::lgg-1::GFP; rol-6(su1006)] were crossed with ced10(n3246) hermaphrodites. Males a from the HZ589 bpIs151 [Psqst-1::sqst-1::GFP; unc-76(+) IV]; him-5(e1490) $V]$ strain, expressing SQST-1::GFP crossed with ced-10 (n3246) hermaphrodites.
N2 (Bristol) was used as the C. elegans wild-type (wt) strain. Hermaphrodites were used throughout of the study.

\section{RNAi Feeding}

For feeding RNAi bacteria, egg lay from gravid adults were directly transferred to NGM plates containing $1 \mathrm{mM}$ of isopropyl $\beta$-D-1-thiogalactopyranoside/ IPTG (referred to as RNAi plates) seeded with $20 \mathrm{X}$ concentrated bacteria containing $50 \mu \mathrm{g} / \mathrm{ml}$ ampicillin, carrying desired plasmid for RNAi of a specific gene (ced-10 or cat-1, depending upon the experiment) or bacteria carrying empty (EV) pL4440 as control and allowed to grow on plates for approximately $50 \mathrm{~h}$ to reach the L4 stage and then another $16 \mathrm{~h}$ for the conduction of actual experiment.

Note: Bacterial clones for RNAi feeding protocol were obtained from the Ahringer library (Source Bioscience, Nottingham, UK) [82] and were then streaked on LBTetracycline-Ampicillin plate which was then incubated at $37{ }^{\circ} \mathrm{C}$. Individual colonies from this freshly streaked plate were grown for $10-12 \mathrm{~h}$ constantly shaking at $37{ }^{\circ} \mathrm{C}$ in LB medium containing $50 \mu \mathrm{g} / \mathrm{ml}$ ampicillin.

\section{Blinding of Experiments and Replicates}

All behavioral studies were completed such that the experimenter was blind to the genotype of the worms. Strains were given letter codes by another member of the laboratory and the code was not broken until all of the replicates for a particular assay were completed. For all assays, we completed a minimum of three biological replicates per strain.

\section{Behavioral Experiments}

Locomotor Rate Assay Locomotor rate assay was performed as described in [33]. Briefly, assay plates were prepared by spreading the $E$. coli strain OP50-1 in a ring on NGM agar [81] in 5-cm petri plates. Assay plates were always freshly spread with bacteria, incubated overnight at $37{ }^{\circ} \mathrm{C}$, and allowed to cool to room temperature before use. Plates for measuring locomotor rate in the absence of bacteria were also incubated at $37^{\circ} \mathrm{C}$. Only synchronized young adult hermaphrodites (16 $\mathrm{h}$ after the late L4 larval stage) were tested. For well-fed animals, locomotor rate was measured by removing 5 animals from plates with ample bacteria, washing the animals twice in $\mathrm{S}$ basal buffer [81], and transferring them to an assay plate in a drop of buffer using a capillary pipette. The drop of buffer used to transfer the animals was absorbed with a Kimwipe. Five minutes after transfer, the number of body bends in $20 \mathrm{~s}$ intervals was sequentially counted for each of 
the five animals on the assay plate and then repeated the same thing for next set of animals in a different assay plate.

Ethanol Avoidance Assay Ethanol avoidance assay was performed as described [34] (Cooper et al. 2015). Synchronized young adult hermaphrodites $(16 \mathrm{~h}$ after the late L4 larval stage) were transferred to assay plates, which are divided into four quadrants: two quadrants seeded with $50 \mu \mathrm{l}$ of $100 \%$ ethanol and the others without. Worms are placed in the center of the assay plate and allowed to move for $30 \mathrm{~min}$ at which point the entire plate is imaged and the worms are scored for their quadrant of preference. Ethanol avoidance is calculated as [(number of worms in control quadrants)-(number of worms in ethanol quadrants)]/total number of worms.

\section{Generation of the Rescue Construct Pced-10:: CFP::ced-10} Pced-10::CFP::ced-10 plasmids were co-injected into worm strain EDC101 and UA196 to generate UA281, baEx167 [Pced-10::CFP::ced-10, rol-6(su1006)]; [Punc54:: $\alpha$-SYN::YFP;ced-10(n3246)] and UA282, baEx167 [Pced-10::CFP::ced-10; rol-6(su1006)]; sid-1(pk3321); baIn33 [Pdat-1::sid-1, Pmyo-2::mCherry]; baIn11[Pdat$1:: \alpha$-SYN; Pdat-1::GFP]). Hermaphrodites were used throughout of the study.

Site-Directed Mutagenesis The construct Pced-10::GFP::ced10 was provided by Erik Lundquist (University of Kansas, Lawrence, KS, USA) as a gift. TagMaster Site-directed mutagenesis (GM Biosciences, Rockville, USA) was used to create mutations (Y66W, Y145F, and M153T) in GFP sequence for changing fluorescence marker as CFP. The product plasmid Pced-10::CFP::ced-10 was sequenced (Eurofins Genomics, Huntsville, AL, USA) to confirm the presence of the desired mutations.

C. elegans Neurodegeneration Assays Worms were analyzed for DA neurodegeneration as described previously [38]. Briefly, 10 L1-stage worms (neuron-specific RNAi worm strain with $\alpha$-Syn UA196 were transferred to the plates (empty vector (EV) and ced-10 RNAi) and grown at $20{ }^{\circ} \mathrm{C}$ until adulthood. Adult worms were then transferred to corresponding freshly made RNAi plates and allowed to lay eggs for $6 \mathrm{~h}$ to synchronize. $\alpha$-SYN-induced DA neurodegeneration was scored at the indicated days after hatching (L4+7; L4 + 3; L4 + 1). To investigate the effect of CED-10 overexpression on $\alpha$-SYN -induced DA degeneration, the strain UA282 was analyzed at L4 +7 days of aging. Worms were considered normal when all six anterior DA neurons (four CEP (cephalic) and two ADE (anterior deirid)) were present without any visible signs of degeneration. If a worm displayed degeneration in at least one of the six neurons, it was scored as exhibiting degeneration. In total, at least 50 adult worms were analyzed for each independent transgenic line or RNAi treatment.

Aggregate Quantification The quantification of aggregates was performed as previously described [83]. Briefly, NL5901animals without and with ced-10(n3246) mutation, together with the EDC101, were age-synchronized by bleaching with $\mathrm{NaOCl}$ and left overnight to hatch. $\mathrm{L} 1$ animals were transferred onto individual NGM plates seeded with Escherichia coli. Aggregates were counted for each animal staged at L $4+5$ days. Images were captured using a Leica SP5 confocal microscope with a $\times 40$ oil immersion lens (HCX PL APO CS). The number of $\alpha$-syn aggregates was determined in the mid body of each animal, taking the vulva position $(\mathrm{V})$ as a reference. Aggregates were defined as discrete, bright structures, with boundaries distinguishable from surrounding fluorescence. Measurements on inclusions were performed using ImageJ software taking into consideration the area dimensions.

Thrashing Assays At L4 + 5 days, animals from the strains N2 wild type, NL5901 [unc-119(ed3) III; pkIs2386 [Punc$54:: \alpha-$ SYN::YFP; unc-119(+)] and EDC01 unc-119(ed3) III; pkIs2386 [Punc-54:: $\alpha$-SYN::YFP; unc-119(+)]; ced-10 (n3246) were placed in a drop of M9 buffer and allowed to recover for $120 \mathrm{~s}$ (to avoid observing behavior associated with stress) after which the number of body bends was counted for $1 \mathrm{~min}$. Movies of swimming worms were recorded using a Leica MZFFLIII stereomicroscope at nominal magnification of 30X and the Hamamatsu ORCA-Flash 4.0LT camera at 17 frames per second (17 fps) for $1 \mathrm{~min}$. Bends per minute were obtained with the Worm Tracker pluggin (wrMTrck), from the ImageJ software [84]. Thirty animals were counted in each experiment unless stated otherwise. Experiments were carried out in triplicate. Statistical analysis was performed using Graphpad Prism version 6.00 for Windows, GraphPad Software, La Jolla, CA, USA).

Detergent Fractionation $\alpha$-SYN oligomeric species were isolated by detergent fractionation as described (Kuwahara et al. 2012) with slight modifications. Briefly, worms were washed three times with M9 buffer, collected as a $100 \mu \mathrm{l}$ pellet, and the pellet was snap-frozen in liquid nitrogen. The pellets were homogenized in $1 \mathrm{~mL}$ of $50 \mathrm{mM}$ Tris- $\mathrm{HCl}$ buffer at $\mathrm{pH} 7.5$ with complete protease inhibitor mixture (Roche Applied Science) by brief sonication. Sonicates were centrifuged two times at $1000 \times g$ for $5 \mathrm{~min}$ to remove debris of worm tissue. The supernatant was then ultracentrifuged at $350,000 \times \mathrm{g}$ for $15 \mathrm{~min}$, and the supernatant was collected as a Tris-HCl soluble fraction. The resulting pellet was subsequently extracted by sonication in $500 \mu \mathrm{l}$ of Triton $\mathrm{X}-100$ (Tris- $\mathrm{HCl}$ buffer with $1 \%$ TritonX-100), $200 \mu \mathrm{l}$ of Sarkosyl (Tris- $\mathrm{HCl}$ with $1 \%$ Sarkosyl), and $100 \mu \mathrm{l}$ of SDS (TrisHCl with SDS sample 
buffer containing $2 \%$ SDS) followed by centrifugation at $350,000 \times \mathrm{g}$ for $15 \mathrm{~min}$. The Tris- $\mathrm{HCl}$ fraction containing $20 \mu \mathrm{g}$ of total proteins, along with equal volumes of Triton $\mathrm{X}-100$, Sarkosyl, and SDS fractions to the Tris- $\mathrm{HCl}$ fraction, were loaded onto the acrylamide gel and separated by SDSPAGE.

Immunoblotting Twenty microliters of protein per strain were loaded from each sample and fraction on Novex NuPAGE 4 $12 \%$ Bis-Tris Gels (Thermo Fisher) an run in MES SDS buffer at $200 \mathrm{~V}$ for $40 \mathrm{~min}$. Resolved proteins were transferred onto $0.45 \mu \mathrm{m}$ nitrocellulose membranes (Amersham) at $200 \mathrm{~mA}$ for $1 \mathrm{~h}$. Blocking was done in 5\% milk powder in PBS for $1 \mathrm{~h}$, followed by overnight incubation at $4{ }^{\circ} \mathrm{C}$ with a mouse monoclonal antibody against $\alpha$-synuclein (1:1000, BD Biosciences, \#610787) diluted in 2\% bovine serum albumin (BSA)/PBS. Incubation with a donkey anti-mouse (1:5000, Amersham) secondary antibody diluted in $1 \%$ milk powder/PBS was performed for $1 \mathrm{~h}$ at room temperature.

Membranes were developed using Supersignal West Femto (Thermo Scientific) in a ImageQuant RT ECL Imager (GE Healthcare).

Autophagy Measurements For measuring the involvement of ced-10(n3246) mutation in autophagy, we followed specific C. elegans protocols [48, 85]. LGG-1::GFP foci were analyzed in the hypodermal seam cells at L3 stage of development. Images were not considered for quantification where the hypodermis was not clear. In total, at least 40 total regions were quantified from 30 different worm samples per genotype.

To confirm the involvement of ced-10 in impairing autophagy, we followed the protocol described in [86]. Pictures of L1 animals (whole animal) and L4 animals (whole intestine), were analyzed by using a $63 \times$ objective. The GFP internal intensity (FI), corresponding to SQST-GFP foci, was quantified at L4 stage of development. The presence of the ced-10 mutation was checked by PCR as described [14]. The presence of the GFP reporter was double checked by PCR using the $g f p$ primers, and under the fluorescence microscope.

Microscopy and Imaging For neurodegeneration assays and aggregates quantification animals were mounted with a coverslip on a $4 \%$ agarose pad in M9, containing $10 \mathrm{mM}$ of sodium azide. Confocal microscopy was performed using a Leica TCS-SP5 confocal spectral microscope (Leica, Barcelona, Spain) and analyzed using ImageJ software (NIH, ver. 1.43, Schindelin, 2015). Animals were examined at $100 \times$ magnification to examine $\alpha-S Y N$ induced DA cell death and at $40 \mathrm{X}$ to examine $\alpha-\mathrm{SYN}$ apparent aggregates.

For autophagy measurements, animals were placed in a $5 \mu \mathrm{L}$ drop of $10 \mathrm{mM}$ solution of levamisole (Sigma-Aldrich, Madrid, Spain). For each independent experiment, between
20 and 30 7-day-old worms of each treatment were examined under a Nikon EclipseTE2000-E epifluorescence microscope equipped with a monochrome camera (Hamamatsu ORCAER) coupled to the Metamorph software (Molecular Devices Corp., Sunnyvale, CA). The system acquires a series of frames at specific Z-axis position (focal plane) using a Z-axis motor device.

\section{Cell Culture Experiments in BE(2)-M17 Neuroblastoma Cell Line}

\section{Cell Culture}

Human neuroblastoma cell line BE(2)-M17 over-expressing wild type $\alpha$-SYN were maintained at $37{ }^{\circ} \mathrm{C}$ and $5 \% \mathrm{CO}_{2}$ in Optimem medium (Gibco) (Thermo-Fisher Scientific, Madrid, Spain) supplemented with $10 \%$ fetal bovine serum (Gibco) (Thermo-Fisher Scientific, Madrid, Spain), $500 \mu \mathrm{g} / \mathrm{ml} \mathrm{G} 418$ (Geneticin) (Sigma-Aldrich, Madrid, Spain) and Penicillin/Streptomycin (Sigma-Aldrich, Madrid, Spain). For immunofluorescence, $2 \times 10^{4}$ cells/well were seeded in 24-well plates on coverslips coated with poly-Dlysine. $\alpha$-SYN aggregation was induced as previously reported ([51] by differentiation with $10 \mu \mathrm{M}$ retinoic acid (SigmaAldrich, Madrid, Spain) for 10 days, followed by treatment with the histone deacetylases inhibitor sodium butyrate (SB) (Sigma-Aldrich, Madrid, Spain) at a concentration of $10 \mathrm{mM}$ for $36 \mathrm{~h}$. To analyze the effect of RAC1 on $\alpha$-SYN aggregation, cells were transduced $24 \mathrm{~h}$ before SB treatment with either the lentiviral empty vector or with the wild type or the constitutively active isoforms of RAC1 at an estimated multiplicity of infection (MOI) of 5 .

\section{Immunofluorescence}

BE(2)-M17 cells were fixed in 4\% paraformaldehyde (PFA) (Sigma-Aldrich, Madrid, Spain) in phosphate saline buffer (PBS) for $30 \mathrm{~min}$ at $4{ }^{\circ} \mathrm{C}$ and permeabilized with Trisbuffered saline (TBS) containing $0.5 \%$ Triton X-100 (Sigma-Aldrich, Madrid, Spain) for $5 \mathrm{~min}$. Cells were then blocked with 3\% normal goat serum (NGS) (Vector Laboratories, Palex Medical, Sant Cugat del Vallès, Spain) in TBS for $1 \mathrm{~h}$ and subsequently incubated with the corresponding primary antibody in $1 \% \mathrm{NGS} / \mathrm{TBS}$ overnight at $4{ }^{\circ} \mathrm{C}$. The following primary antibodies were used: mouse anti- $\alpha$-SYN (610,787; 1:500) (BD Biosciences, Madrid, Spain), rabbit anti- $\alpha$-SYN (2642; 1:500) (Cell Signaling, Leiden, The Netherlands). Incubation with goat secondary antibodies conjugated with Alexa488 (anti-mouse, A11001; or Alexa594 (anti-mouse, A11005; anti-rabbit, A11012) (Thermo-Fisher Scientific, Madrid, Spain) was done in $1 \%$ NGS/TBS for $1 \mathrm{~h}$ at RT. Between each incubation period cells were rinsed three times in TBS. 


\section{Thioflavin S Staining}

After incubation with the antibodies, coverslips were immersed in $0.005 \%$ thioflavin S (Sigma-Aldrich, Madrid, Spain) in PBS for 8 min and then rinsed twice in ethanol $70 \%$ and once in PBS. Only after thioflavin S staining, nuclei were counterstained with Hoechst 33342 (Thermo Fisher Scientific, Madrid, Spain, 1:10,000 in PBS) for $10 \mathrm{~min}$.

\section{Microscopy and IF Quantification}

Cells were cover slipped using Dako Cytomation Fluorescent Mounting Medium (Dako, Sant Just Desvern, Spain). Immunofluorescence images were acquired using standard filter sets using an Olympus FluoView TM FV1000 confocal microscope and the FV10_ASW 4.2 visualization software.

$\alpha$-SYN fluorescence intensity for each condition was analyzed in an average of 150 cells from 6 different random fields at an objective magnification of $40 \times$. Quantification of Thioflavin S positive aggregates in each condition was analyzed in an average of 20 cells from 3 different random fields by measuring the fluorescent area per cell. All quantification analyses were done using ImageJ software (NIH, USA).

\section{Cell Culture Experiments with iPSC Cell Lines Derived from Human Patients}

Previously generated iPSC lines SP-11.1 (from control) and SP-12.3 (from patients with familial PD with the LRRK2 G2019S mutation) were used and culture and differentiation were carried out as described, (34), following a protocol approved by the Spanish competent authorities (Commission on Guarantees concerning the Donation and Use of Human Tissues and Cells of the Carlos III Health Institute). Briefly, hiPSC were cultured on matrigel (Corning Limited, Life Sciences, UK) and maintained in hESC medium, consisting on KO-DMEM (Invitrogen, Thermo Fisher Scientific, Madrid, Spain) supplemented with 20\% KO-Serum Replacement (Invitrogen, Thermo Fisher Scientific, Madrid, Spain), 2 mM Glutamax (Invitrogen, Thermo Fisher Scientific, Madrid, Spain), $50 \mu \mathrm{M}$ 2-mercaptoethanol (Invitrogen, Thermo Fisher Scientific, Madrid, Spain), nonessential aminoacids (Cambrex, Nottingham, UK) and $10 \mathrm{ng} / \mathrm{ml}$ bFGF (Peprotech, London, UK). Medium was preconditioned overnight by irradiated mouse embryonic fibroblast and hiPSC were maintained on Matrigel (Corning Limited, Life Sciences, UK) at $37{ }^{\circ} \mathrm{C}, 5 \% \mathrm{CO} 2$.

For DAn differentiation, iPSC were transduced with LV.NES.LMX1A.GFP and processed as previously described (79). Briefly, confluent iPSC $10 \mathrm{~cm}$ dishes were disaggregated with accutase and embryoid bodies (EB) were generated using forced aggregation method in V-shaped 96 well plates. Two days later, EBs were patterned as ventral midbrain by culturing them in suspension for 10 days in N2B27 supplemented with $100 \mathrm{ng} / \mathrm{ml} \mathrm{SHH}$ (Peprotech, London, UK), $100 \mathrm{ng} / \mathrm{ml} \mathrm{FGF8} \mathrm{(Peprotech,} \mathrm{London,} \mathrm{UK)} \mathrm{and} 10 \mathrm{ng} / \mathrm{ml}$ FGF2 (Peprotech, London, UK). Then, for $\alpha$-SYN and neurite analysis, differentiation to midbrain DAn was performed on the top of PA6 murine stromal cells for 3 weeks (79). To analyze $\alpha$-SYN levels, neuronal cultures were gently trypsinized and re-plated in matrigel-coated slides for 3 days, then transduced with the two different RAC1 isoforms or the control LV, at an estimated MOI of 10. Cells were fixed and analyzed 7 days after transduction. For long-term culture studies, EBs were mechanically dissociated by repeated pipetting after the induction step. Salient EB fragments were transduced with the two different RAC1 isoforms or the control LV at an estimated MOI of 3. Three days post-transduction, the aggregates were seeded on primary murine astrocytes and cultured for 9 weeks.

\section{Lentiviral Vectors}

RAC1 wild type (WT) and constitutively active (V12) (CA) forms were amplified by means of PCR from expression plasmids kindly provided by (Dr Francisco Sánchez-Madrid; Spanish National Center for Cardiovascular Research (CNIC), Madrid, Spain). Subsequently, RAC1-(WT) and RAC1-(CA) cDNA were cloned under the human phosphoglycerate kinase (PGK) promoter of pCCL.cPPT-hPGKIRES.eGFP-WPRE lentiviral transfer vector. High-titer vesicular stomatitis virus (VSV)-pseudotyped LV stocks were produced in 293 T cells by calcium phosphate-mediated transient transfection of the transfer vector, the late generation packaging construct pMDL, and the VSV envelope-expressing construct pMD2.G, and purified by ultracentrifugation as previously described [87]. Expression titers, determined on HeLa cells by fluorescence-activated cell sorter (FACS) analysis (FACSCalibur, Becton Dickinson), were as follows: LVRAC1 (WT): $2.60 \cdot 10^{\wedge} 8 \mathrm{TU} / \mathrm{mL}$; LV-RAC1 (CA): 2.24 • $10^{\wedge} 8 \mathrm{TU} / \mathrm{mL}$; LV-PGK-GFP: $5.08 \cdot 10^{\wedge} 9 \mathrm{TU} / \mathrm{mL}$.

\section{Immunofluorescence for iPSC-Derived Cells}

iPSC-derived cells were fixed with $4 \%$ paraformaldehyde (PFA) in Tris-buffered saline (TBS) buffer for $20 \mathrm{~min}$ and blocked in $0.3 \%$ Triton X-100 (Sigma-Aldrich, Madrid, Spain) with 3\% donkey serum for $2 \mathrm{~h}$. In the case of $\alpha$-SYN and LC3 staining, Triton X-100 was kept at $0.01 \%$ for the blocking and antibody incubation steps. The following primary antibodies were used: mouse anti- $\alpha$-SYN $(610787 ; 1: 500)$ (BD Biosciences, Madrid, Spain), rabbit anti-TH (sc-14007; 1:500) (Santa Cruz Biotechnology, Madrid, Spain), chicken anti-GFP (1020; 1:250) from Aves Labs (Cosmo Bio, AbBcn S.L., Bellaterra, Spain), rabbit anti-cleaved Caspase3 (9664; 1:400) (Cell Signaling) and LC3B (2775; 1:100) (Cell 
Signaling). Images were acquired using a Leica SP5 confocal microscope.

\section{a-SYN and Neurite Analysis}

$\alpha-\mathrm{SYN}$ and neurite analysis were performed after a total of 4 weeks of differentiation plus 1 week after LV transduction on iPSC-derived DA neurons. DA neurons were randomly selected, using a Leica SP5 confocal microscope, and analyzed with ImageJ for $\alpha$-SYN intensity levels and with the ImageJ plugin NeuronJ to determine the number of neurites per cell, number of terminals and branch points.

\section{Cleaved Caspase-3, Apoptotic Cell Number and Autophagosome Accumulation and Analysis}

Cleaved caspase-3, apoptotic cell number and autophagosome accumulation analysis were performed after a total of 9 weeks of differentiation on iPSC-derived DAn grown on murine astrocytes. DA neurons were randomly selected, using a Leica SP5 confocal microscope, and analyzed with ImageJ to determine the fraction of the neuronal soma area stained by cleaved caspase-3 or covered by LC3-positive particles.

\section{Statistical Analysis}

All experiments were performed at least three independent times. All data are presented as mean \pm SEM. Group means were compared with either the Student's $t$ test or ANOVA. All $P$ values were two tailed, and a $P$ value of less than 0.05 was considered statistically significant. All statistical analyses were analyzed using GraphPad Prism (San Diego, CA, USA) software.

Outlier values were identified with the Grubbs' tests and excluded from the analysis.

Differences among means were analyzed either by 1 - or 2 way analysis of variance (ANOVA), as appropriate, using the post hoc Tukey's multiple comparison test. In all cases, the null hypothesis was rejected at the 0.05 level.

\section{Biological Enrichment Analysis}

Transcriptomic analysis of iPSC-derived DAn from PD patients $(n=10)$ and healthy controls $(n=4)$ was done as previously described [54]. Identified differentially expressed genes (DEGs) after multiple testing adjustment of $P$ values $(n=437)$ were subjected to biological enrichment analysis. To this end, we used the Core Analysis module of the Ingenuity ${ }^{\circledR}$ Pathway Analysis (IPA) software (Qiagen, Redwood City, www. qiagen.com/ingenuity) to identify biological enrichment of canonical pathways deregulated in iPSC-derived DAn from PD patients. More specifically, we used the Ingenuity Knowledge Base of Genes, and considered only direct molecules and/or relationships for humans. Statistical significance of canonical pathways was computed by using the Fischer's exact test and significance levels were set at $\mathrm{P}$ below 0.05 . Using IPA we also calculated Z-score values which consider the directional effect of one molecule on another molecule or on a process, and also the direction of change of molecules in the dataset and provide predictions about whether the pathway is predicted to be activated or inhibited, or if the pathway is ineligible for such an assessment.

Acknowledgements The genetic strain BR3579 was kindly provided by Dr. Ralf Baumeister (Albert-Ludwing University, Freiburg/Brisgau, Germany). Plasmids Rac1-GFP WT and Rac1-GFP (CA) HYM772 were kindly provided by Dr. Francisco Sánchez-Madrid (Spanish National Center for Cardiovascular Research (CNIC), Madrid, Spain). The construct Pced-10::CFP::CED-10 was a generous gift provided by Erik Lundquist (University of Kansas, Lawrence, KS, USA). Human neuroblastoma cell line BE(2)-M17 over-expressing wild type $\alpha$-SYN was provided by Dr. B. Wolozin (Boston University School of Medicine).

Funding This work was supported by the following grants: Instituto de Salud Carlos III (PI15/01255), co-funded by European Union (ERDF/ ESF, "investing in your future"; Spanish Ministry of Economy and Competitiveness (MINECO) (BFU2015-64408-P) and the Instituto de Salud Carlos III (PI11/00072, co-financed by the Fondo Social Europeo) to AMV; Spanish Ministry of Economy and Competitiveness (MINECO) (SAF2016-77541-R) to MV; (SAF2015-69706-R) and Instituto de Salud Carlos III (ISCIII)/FEDER (Red de Terapia CelularTerCel RD16/0011/0024) to AR; and (BFU2016-80870-P) to AC. This work was also supported by the European Research Council (ERC) 2012StG (311736-PD-HUMMODEL) to AC. The authors also thank the CERCA Program from the Generalitat de Catalunya and AGAUR (2014-SGR-1460). ED and AV are members of the GENIE and EUROS Cost Action of the European Union. CC was partially supported by pre-doctoral fellowships from the Spanish Ministry of EducationMEC (FPU12/03332).

ED and AV are members of the GENIE and EU-ROS Cost Action of the European Union.

Compliance with Ethical Standards All experiments were performed under the guidelines and protocols of the Ethical Committee for Animal Experimentation (CEEA) of the University of Barcelona. All procedures adhered to internal and EU guidelines for research involving derivation of pluripotent cell lines. All subjects gave informed consent for the study using forms approved by the Ethical Committee on the Use of Human Subjects in Research at Hospital Clinic in Barcelona, Spain. Generation of iPSC lines was approved by the Advisory Committee for Human Tissue and Cell Donation and Use, by the Commission on Guarantees concerning the Donation and Use of Human Tissues and Cells of the Carlos III Health Institute, Madrid, Spain. All procedures were done in accordance with institutional guidelines following the Spanish legislation.

Conflict of Interest The authors declare that they have no conflict of interest.

Open Access This article is distributed under the terms of the Creative Commons Attribution 4.0 International License (http:// creativecommons.org/licenses/by/4.0/), which permits use, duplication, adaptation, distribution and reproduction in any medium or format, as long as you give appropriate credit to the original author(s) and the source, provide a link to the Creative Commons license, and indicate if changes were made. 


\section{References}

1. Michel PP, Hirsch EC, Hunot S (2016) Understanding dopaminergic cell death pathways in Parkinson disease. Neuron 90(4):675691. https://doi.org/10.1016/j.neuron.2016.03.038

2. Dehay B, Bourdenx M, Gorry P, Przedborski S, Vila M, Hunot S, Singleton A, Olanow CW et al (2015) Targeting $\alpha$-synuclein for treatment of Parkinson's disease: mechanistic and therapeutic considerations. Lancet Neurol 14(8):855-866. https://doi.org/10.1016/ S1474-4422(15)00006-X

3. Weinreb PH, Zhen W, Poon AW, Conway KA, Lansbury PT (1996) NACP, a protein implicated in Alzheimer's disease and learning, is natively unfolded. Biochemistry 35(43):13709-13715. https://doi. org/10.1021/bi961799n

4. Iwai A, Masliah E, Yoshimoto M, Ge N, Flanagan L, Rohan de Silva HA, Kittel A, Saitoh T (1995) The precursor protein of non-A beta component of Alzheimer's disease amyloid is a presynaptic protein of the central nervous system. Neuron 14(2):467-475. https://doi.org/10.1016/0896-6273(95)90302-X

5. Wong YC, Krainc D (2017) $\alpha$-synuclein toxicity in neurodegeneration: mechanism and therapeutic strategies. Nat Med 23(2):1-13. https://doi.org/10.1038/nm.4269

6. Winner B, Jappelli R, Maji SK, Desplats PA, Boyer L, Aigner S, Hetzer C, Loher T et al (2011) In vivo demonstration that alphasynuclein oligomers are toxic. Proc Natl Acad Sci U S A 108(10): 4194 4199. https://doi.org/10.1073/pnas.1100976108

7. Hall A, Lalli G (2010) Rho and Ras GTPases in axon growth, guidance, and branching. Cold Spring Harb Perspect Biol 2(2): a001818. https://doi.org/10.1101/cshperspect.a001818

8. Musilli M, Ciotti MT, Pieri M, Martino A, Borrelli S, Dinallo V, Diana G (2016) Therapeutic effects of the Rho GTPase modulator CNF1 in a model of Parkinson's disease. Neuropharmacology 109: 357-365. https://doi.org/10.1016/j.neuropharm.2016.06.016

9. Schnack C, Danzer KM, Hengerer B, Gillardon F (2008) Protein array analysis of oligomerization-induced changes in alphasynuclein protein-protein interactions points to an interference with Cdc42 effector proteins. Neuroscience 154(4):1450-1457. https:// doi.org/10.1016/j.neuroscience.2008.02.049

10. Koch JC, Bitow F, Haack J, d'Hedouville Z, Zhang JN, Tönges L, Michel U, Oliveira LMA et al (2015) Alpha-Synuclein affects neurite morphology, autophagy, vesicle transport and axonal degeneration in CNS neurons. Cell Death Dis 6(7):e1811. https://doi.org/ 10.1038/cddis.2015.169

11. Prots I, Veber V, Brey S, Campioni S, Buder K, Riek R, Böhm KJ, Winner B (2013) $\alpha$-Synuclein oligomers impair neuronal microtubule-kinesin interplay. J Biol Chem 288(30):2174221754. https://doi.org/10.1074/jbc.M113.451815

12. Kinchen JM, Cabello J, Klingele D, Wong K, Feichtinger R, Schnabel H, Schnabel R, Hengartner MO (2005) Two pathways converge at CED-10 to mediate actin rearrangement and corpse removal in C. elegans. Nature 434(7029):93-99. https://doi.org/ 10.1038/nature 03263

13. Hou ST, Jiang SX, Smith RA (2008) Permissive and repulsive cues and signalling pathways of axonal outgrowth and regeneration. Int Rev Cell Mol Biol 267:125-181. https://doi.org/10.1016/S19376448(08)00603-5

14. Cabello J et al (2014) PDR-1/hParkin negatively regulates the phagocytosis of apoptotic cell corpses in Caenorhabditis elegans. Cell Death Dis 5

15. Paisán-Ruíz C et al (2004) Cloning of the gene containing mutations that cause PARK8-linked Parkinson's disease. Neuron 44(4): 595-600. https://doi.org/10.1016/j.neuron.2004.10.023

16. Chan D, Citro A, Cordy JM, Shen GC, Wolozin B (2011) Rac1 protein rescues neurite retraction caused by G2019S leucine-rich repeat kinase 2 (LRRK2). J Biol Chem 286(18):16140-16149. https://doi.org/10.1074/jbc.M111.234005

17. Pesaresi MG, Amori I, Giorgi C, Ferri A, Fiorenzo P, Gabanella F, Salvatore AM, Giorgio M et al (2011) Mitochondrial redox signalling by p66Shc mediates ALS-like disease through Rac1 inactivation. Hum Mol Genet 20(21):4196-4208. https://doi.org/10.1093/ $\mathrm{hmg} / \mathrm{ddr} 347$

18. Philippens IH et al (2013) Oral treatment with the NADPH oxidase antagonist apocynin mitigates clinical and pathological features of parkinsonism in the MPTP marmoset model. J NeuroImmune Pharmacol 8(3):715-726. https://doi.org/10.1007/s11481-0139450-z

19. Vogiatzi T, Xilouri M, Vekrellis K, Stefanis L (2008) Wild type alpha-synuclein is degraded by chaperone-mediated autophagy and macroautophagy in neuronal cells. J Biol Chem 283(35): 23542-23556. https://doi.org/10.1074/jbc.M801992200

20. Sánchez-Danés A, Richaud-Patin Y, Carballo-Carbajal I, JiménezDelgado S, Caig C, Mora S, di Guglielmo C, Ezquerra M et al (2012) Disease-specific phenotypes in dopamine neurons from human iPS-based models of genetic and sporadic Parkinson's disease. EMBO Mol Med 4(5):380-395. https://doi.org/10.1002/emmm. 201200215

21. Zavodszky E et al (2014) Mutation in VPS35 associated with Parkinson's disease impairs WASH complex association and inhibits autophagy. Nat Commun 5:3828

22. Huang Y, Chegini F, Chua G, Murphy K, Gai W, Halliday GM (2012) Macroautophagy in sporadic and the genetic form of Parkinson's disease with the A53T $\alpha$-synuclein mutation. Transl Neurodegener 1(1):2. https://doi.org/10.1186/2047-9158-1-2

23. Ramirez A, Heimbach A, Gründemann J, Stiller B, Hampshire D, Cid LP, Goebel I, Mubaidin AF et al (2006) Hereditary parkinsonism with dementia is caused by mutations in ATP13A2, encoding a lysosomal type 5 P-type ATPase. Nat Genet 38(10):1184-1191. https://doi.org/10.1038/ng1884

24. Schapira AH (2015) Glucocerebrosidase and Parkinson disease: recent advances. Mol Cell Neurosci 66(Pt A):37-42

25. Qu X, Zou Z, Sun Q, Luby-Phelps K, Cheng P, Hogan RN, Gilpin C, Levine B (2007) Autophagy gene-dependent clearance of apoptotic cells during embryonic development. Cell 128(5):931-946. https://doi.org/10.1016/j.cell.2006.12.044

26. Li W, Zou W, Yang Y, Chai Y, Chen B, Cheng S, Tian D, Wang X et al (2012) Autophagy genes function sequentially to promote apoptotic cell corpse degradation in the engulfing cell. J Cell Biol 197(1):27-35. https://doi.org/10.1083/jcb.201111053

27. Cheng S, Wu Y, Lu Q, Yan J, Zhang H, Wang X (2013) Autophagy genes coordinate with the class II PI/PtdIns 3-kinase PIKI-1 to regulate apoptotic cell clearance in C. elegans. Autophagy 9(12): 2022-2032. https://doi.org/10.4161/auto.26323

28. Reddien PW, Horvitz HR (2000) CED-2/CrkII and CED-10/Rac control phagocytosis and cell migration in Caenorhabditis elegans. Nat Cell Biol 2(3):131-136. https://doi.org/10.1038/35004000

29. Shakir MA, Gill JS, Lundquist EA (2006) Interactions of UNC-34 enabled with Rac GTPases and the NIK kinase MIG-15 in Caenorhabditis elegans axon pathfinding and neuronal migration. Genetics 172(2):893-913. https://doi.org/10.1534/genetics.105. 046359

30. Ezcurra M, Tanizawa Y, Swoboda P, Schafer WR (2011) Food sensitizes $C$. elegans avoidance behaviours through acute dopamine signalling. EMBO J 30(6):1110-1122. https://doi.org/10.1038/ emboj.2011.22

31. Hills T, Brockie PJ, Maricq AV (2004) Dopamine and glutamate control area-restricted search behavior in Caenorhabditis elegans. J Neurosci 24(5):1217-1225. https://doi.org/10.1523/JNEUROSCI. 1569-03.2004

32. Sanyal S, Wintle RF, Kindt KS, Nuttley WM, Arvan R, Fitzmaurice P, Bigras E, Merz DC et al (2004) Dopamine modulates the 
plasticity of mechanosensory responses in Caenorhabditis elegans. EMBO J 23(2):473-482. https://doi.org/10.1038/sj.emboj.7600057

33. Sawin ER, Ranganathan R, Horvitz HR (2000) C. elegans locomotory rate is modulated by the environment through a dopaminergic pathway and by experience through a serotonergic pathway. Neuron 26(3):619-631. https://doi.org/10.1016/S0896-6273(00) 81199-X

34. Lee J, Jee C, McIntire SL (2009) Ethanol preference in C. elegans. Genes Brain Behav 8(6):578-585. https://doi.org/10.1111/j.1601183X.2009.00513.x

35. Hamamichi S, Rivas RN, Knight AL, Cao S, Caldwell KA, Caldwell GA (2008) Hypothesis-based RNAi screening identifies neuroprotective genes in a Parkinson's disease model. Proc Natl Acad Sci U S A 105(2):728-733. https://doi.org/10.1073/pnas. 0711018105

36. Harrington AJ, Hamamichi S, Caldwell GA, Caldwell KA (2010) C. elegans as a model organism to investigate molecular pathways involved with Parkinson's disease. Dev Dyn 239(5):1282-1295. https://doi.org/10.1002/dvdy.22231

37. Sulston J, Dew M, Brenner S (1975) Dopaminergic neurons in the nematode Caenorhabditis elegans. J Comp Neurol 163(2):215-226. https://doi.org/10.1002/cne.901630207

38. Cao S, Gelwix CC, Caldwell KA, Caldwell GA (2005) Torsinmediated protection from cellular stress in the dopaminergic neurons of Caenorhabditis elegans. J Neurosci 25(15):3801-3812. https://doi.org/10.1523/JNEUROSCI.5157-04.2005

39. Hochreiter-Hufford A, Ravichandran KS (2013) Clearing the dead: apoptotic cell sensing, recognition, engulfment, and digestion. Cold Spring Harb Perspect Biol 5(1):a008748. https://doi.org/10.1101/ cshperspect.a008748

40. Oczypok EA, Oury TD, Chu CT (2013) It's a cell-eat-cell world: autophagy and phagocytosis. Am J Pathol 182(3):612-622. https:// doi.org/10.1016/j.ajpath.2012.12.017

41. van Ham TJ, Thijssen KL, Breitling R, Hofstra RM, Plasterk RH, Nollen EA (2008) C. elegans model identifies genetic modifiers of alpha-synuclein inclusion formation during aging. PLoS Genet 4(3):e1000027. https://doi.org/10.1371/journal.pgen.1000027

42. Martinez BA, Kim H, Ray A, Caldwell GA, Caldwell KA (2015) A bacterial metabolite induces glutathione-tractable proteostatic damage, proteasomal disturbances, and PINK1-dependent autophagy in C. elegans. Cell Death Dis 6(10):e1908. https://doi.org/10.1038/ cddis. 2015.270

43. van der Goot AT, Zhu W, Vazquez-Manrique RP, Seinstra RI, Dettmer K, Michels H, Farina F, Krijnen J et al (2012) Delaying aging and the aging-associated decline in protein homeostasis by inhibition of tryptophan degradation. Proc Natl Acad Sci U S A 109(37):14912-14917. https://doi.org/10.1073/pnas.1203083109

44. Gidalevitz T, Krupinski T, Garcia S, Morimoto RI (2009) Destabilizing protein polymorphisms in the genetic background direct phenotypic expression of mutant SOD1 toxicity. PLoS Genet 5(3):e1000399. https://doi.org/10.1371/journal.pgen. 1000399

45. Kuwahara T, Tonegawa R, Ito G, Mitani S, Iwatsubo T (2012) Phosphorylation of $\alpha$-synuclein protein at Ser-129 reduces neuronal dysfunction by lowering its membrane binding property in Caenorhabditis elegans. J Biol Chem 287(10):7098-7109. https:// doi.org/10.1074/jbc.M111.237131

46. Cuervo AM, Stefanis L, Fredenburg R, Lansbury PT, Sulzer D (2004) Impaired degradation of mutant alpha-synuclein by chaperone-mediated autophagy. Science 305(5688):1292-1295. https://doi.org/10.1126/science.1101738

47. Büttner S, Broeskamp F, Sommer C, Markaki M, Habernig L, Alavian-Ghavanini A, Carmona-Gutierrez D, Eisenberg T et al (2014) Spermidine protects against $\alpha$-synuclein neurotoxicity. Cell Cycle 13(24):3903-3908. https://doi.org/10.4161/15384101. 2014.973309
48. Zhang H, Chang JT, Guo B, Hansen M, Jia K, Kovács AL, Kumsta C, Lapierre LR et al (2015) Guidelines for monitoring autophagy in Caenorhabditis elegans. Autophagy 11(1):9-27. https://doi.org/10. 1080/15548627.2014.1003478

49. Guo B, Huang X, Zhang P, Qi L, Liang Q, Zhang X, Huang J, Fang B et al (2014) Genome-wide screen identifies signaling pathways that regulate autophagy during Caenorhabditis elegans development. EMBO Rep 15(6):705-713. https://doi.org/10.1002/embr. 201338310

50. Tian Y, Li Z, Hu W, Ren H, Tian E, Zhao Y, Lu Q, Huang X et al (2010) C. elegans screen identifies autophagy genes specific to multicellular organisms. Cell 141(6):1042-1055. https://doi.org/ 10.1016/j.cell.2010.04.034

51. Jiang P, Gan M, Ebrahim A, Lin WL, Melrose HL, Yen SHC (2010) ER stress response plays an important role in aggregation of $\alpha$ synuclein. Mol Neurodegener 5(1):56. https://doi.org/10.1186/ 1750-1326-5-56

52. Raiss CC, Braun TS, Konings IBM, Grabmayr H, Hassink GC, Sidhu A, le Feber J, Bausch AR et al (2016) Functionally different $\alpha$-synuclein inclusions yield insight into Parkinson's disease pathology. Sci Rep 6(1):23116. https://doi.org/10.1038/srep23116

53. MacLeod D, Dowman J, Hammond R, Leete T, Inoue K, Abeliovich A (2006) The familial parkinsonism gene LRRK2 regulates neurite process morphology. Neuron 52(4):587-593. https:// doi.org/10.1016/j.neuron.2006.10.008

54. Fernández-Santiago R et al (2015) Aberrant epigenome in iPSCderived dopaminergic neurons from Parkinson's disease patients. EMBO Mol Med 7(12):1529-1546. https://doi.org/10.15252/ emmm.201505439

55. Johnson MA, Weick JP, Pearce RA, Zhang SC (2007) Functional neural development from human embryonic stem cells: accelerated synaptic activity via astrocyte coculture. J Neurosci 27(12):30693077. https://doi.org/10.1523/JNEUROSCI.4562-06.2007

56. Tahirovic S, Hellal F, Neukirchen D, Hindges R, Garvalov BK, Flynn KC, Stradal TE, Chrostek-Grashoff A et al (2010) Rac1 regulates neuronal polarization through the WAVE complex. J Neurosci 30(20):6930-6943. https://doi.org/10.1523/ JNEUROSCI.5395-09.2010

57. Lorenzetto E, Ettorre M, Pontelli V, Bolomini-Vittori M, Bolognin S, Zorzan S, Laudanna C, Buffelli M (2013) Rac1 selective activation improves retina ganglion cell survival and regeneration. PLoS One 8(5):e64350. https://doi.org/10.1371/journal.pone.0064350

58. Kanekura K, Hashimoto Y, Kita Y, Sasabe J, Aiso S, Nishimoto I, Matsuoka M (2005) A Rac1/phosphatidylinositol 3-kinase/Akt3 anti-apoptotic pathway, triggered by AlsinLF, the product of the ALS2 gene, antagonizes $\mathrm{Cu} / \mathrm{Zn}$-superoxide dismutase (SOD1) mutant-induced motoneuronal cell death. J Biol Chem 280(6): 4532-4543. https://doi.org/10.1074/jbc.M410508200

59. Stankiewicz TR, Linseman DA (2014) Rho family GTPases: key players in neuronal development, neuronal survival, and neurodegeneration. Front Cell Neurosci 8:314

60. Nichols AL et al (2016) The apoptotic engulfment machinery regulates axonal degeneration in C. elegans neurons. Cell Rep 14(7): 1673-1683. https://doi.org/10.1016/j.celrep.2016.01.050

61. Zhang B, Zhang Y, Shacter E (2004) Rac1 inhibits apoptosis in human lymphoma cells by stimulating bad phosphorylation on Ser-75. Mol Cell Biol 24(14):6205-6214. https://doi.org/10.1128/ MCB.24.14.6205-6214.2004

62. Cheng C, Kong X, Wang H, Gan H, Hao Y, Zou W, Wu J, Chi Y et al (2009) Trihydrophobin 1 interacts with PAK1 and regulates ERK/MAPK activation and cell migration. J Biol Chem 284(13): 8786-8796. https://doi.org/10.1074/jbc.M806144200

63. Cicenas J (2008) The potential role of Akt phosphorylation in human cancers. Int J Biol Markers 23(1):1-9. https://doi.org/10.5301/ JBM.2008.618 
64. Nagarajan A, Ning Y, Reisner K, Buraei Z, Larsen JP, Hobert O, Doitsidou M (2014) Progressive degeneration of dopaminergic neurons through TRP channel-induced cell death. J Neurosci 34(17): 5738-5746. https://doi.org/10.1523/JNEUROSCI.4540-13.2014

65. Coles CH, Bradke F (2015) Coordinating neuronal actinmicrotubule dynamics. Curr Biol 25(15):R677-R691. https://doi. org/10.1016/j.cub.2015.06.020

66. Chia JX, Efimova N, Svitkina TM (2016) Neurite outgrowth is driven by actin polymerization even in the presence of actin polymerization inhibitors. Mol Biol Cell 27(23):3695-3704. https://doi. org/10.1091/mbc.E16-04-0253

67. Dubey J, Ratnakaran N, Koushika SP (2015) Neurodegeneration and microtubule dynamics: death by a thousand cuts. Front Cell Neurosci 9:343

68. Godena VK, Brookes-Hocking N, Moller A, Shaw G, Oswald M, Sancho RM, Miller CCJ, Whitworth AJ et al (2014) Increasing microtubule acetylation rescues axonal transport and locomotor deficits caused by LRRK2 Roc-COR domain mutations. Nat Commun 5:5245. https://doi.org/10.1038/ncomms6245

69. Gandhi PN, Wang X, Zhu X, Chen SG, Wilson-Delfosse AL (2008) The Roc domain of leucine-rich repeat kinase 2 is sufficient for interaction with microtubules. J Neurosci Res 86(8):1711-1720. https://doi.org/10.1002/jnr.21622

70. Okada T, Hirai C, Badawy SMM, Zhang L, Kajimoto T, Nakamura $\mathrm{Si}$ (2016) Impairment of PDGF-induced chemotaxis by extracellular $\alpha$-synuclein through selective inhibition of Rac1 activation. Sci Rep 6(1):37810. https://doi.org/10.1038/srep37810

71. Sousa VL, Bellani S, Giannandrea M, Yousuf M, Valtorta F, Meldolesi J, Chieregatti E (2009) \{alpha\}-synuclein and its A30P mutant affect actin cytoskeletal structure and dynamics. Mol Biol Cell 20(16):3725-3739. https://doi.org/10.1091/mbc.E08-03-0302

72. Crews L, Spencer B, Desplats P, Patrick C, Paulino A, Rockenstein E, Hansen L, Adame A et al (2010) Selective molecular alterations in the autophagy pathway in patients with Lewy body disease and in models of alpha-synucleinopathy. PLoS One 5(2):e9313. https:// doi.org/10.1371/journal.pone.0009313

73. Mak SK, McCormack AL, Manning-Boğ AB, Cuervo AM, di Monte DA (2010) Lysosomal degradation of alpha-synuclein in vivo. J Biol Chem 285(18):13621-13629. https://doi.org/10. 1074/jbc.M109.074617

74. Vijayakumaran S, Wong M, Antony H, Pountney D (2015) Direct and/or indirect roles for SUMO in modulating alpha-synuclein toxicity. Biomol Ther 5(3):1697-1716. https://doi.org/10.3390/ biom5031697

75. Ahmed I, Liang Y, Schools S, Dawson VL, Dawson TM, Savitt JM (2012) Development and characterization of a new Parkinson's disease model resulting from impaired autophagy. J Neurosci 32(46): 16503-16509. https://doi.org/10.1523/JNEUROSCI.0209-12.2012
76. Ebrahimi-Fakhari D, Cantuti-Castelvetri I, Fan Z, Rockenstein E, Masliah E, Hyman BT, McLean PJ, Unni VK (2011) Distinct roles in vivo for the ubiquitin-proteasome system and the autophagylysosomal pathway in the degradation of $\alpha$-synuclein. J Neurosci 31(41):14508-14520. https://doi.org/10.1523/JNEUROSCI.156011.2011

77. Winslow AR, Chen CW, Corrochano S, Acevedo-Arozena A, Gordon DE, Peden AA, Lichtenberg M, Menzies FM et al (2010) $\alpha$-Synuclein impairs macroautophagy: implications for Parkinson's disease. J Cell Biol 190(6):1023-1037. https://doi.org/10.1083/jcb. 201003122

78. Orenstein SJ, Kuo SH, Tasset I, Arias E, Koga H, Fernandez-Carasa I, Cortes E, Honig LS et al (2013) Interplay of LRRK2 with chaperone-mediated autophagy. Nat Neurosci 16(4):394-406. https://doi.org/10.1038/nn.3350

79. Alegre-Abarrategui J, Christian H, Lufino MMP, Mutihac R, Venda LL, Ansorge O, Wade-Martins R (2009) LRRK2 regulates autophagic activity and localizes to specific membrane microdomains in a novel human genomic reporter cellular model. Hum Mol Genet 18(21):4022-4034. https://doi.org/10.1093/hmg/ddp346

80. Saha S, Liu-Yesucevitz L, Wolozin B (2014) Regulation of autophagy by LRRK2 in Caenorhabditis elegans. Neurodegener Dis 13(23):110-113. https://doi.org/10.1159/000355654

81. Brenner S (1974) The genetics of Caenorhabditis elegans. Genetics 77(1):71-94

82. Kamath RS, Ahringer J (2003) Genome-wide RNAi screening in Caenorhabditis elegans. Methods 30(4):313-321. https://doi.org/ 10.1016/S1046-2023(03)00050-1

83. Nollen EA et al (2004) Genome-wide RNA interference screen identifies previously undescribed regulators of polyglutamine aggregation. Proc Natl Acad Sci U S A 101(17):6403-6408. https:// doi.org/10.1073/pnas.0307697101

84. Schindelin J, Rueden CT, Hiner MC, Eliceiri KW (2015) The ImageJ ecosystem: an open platform for biomedical image analysis. Mol Reprod Dev 82(7-8):518-529. https://doi.org/10.1002/mrd. 22489

85. Palmisano NJ, Meléndez A (2016) Detection of autophagy in Caenorhabditis elegans. Cold Spring Harb Protoc 2016(2): pdb.top070466. https://doi.org/10.1101/pdb.top070466

86. Lapierre LR et al (2013) The TFEB orthologue HLH-30 regulates autophagy and modulates longevity in Caenorhabditis elegans. Nat Commun 4:2267

87. Consiglio A, Gritti A, Dolcetta D, Follenzi A, Bordignon C, Gage FH, Vescovi AL, Naldini L (2004) Robust in vivo gene transfer into adult mammalian neural stem cells by lentiviral vectors. Proc Natl Acad Sci U S A 101(41):14835-14840. https://doi.org/10.1073/ pnas. 0404180101 